\title{
Regio- and Enantioselective Intermolecular Rhodium-Catalyzed [2+2+2] Carbocyclization Reactions of 1,6-Enynes with Methyl Arylpropiolates
}

\author{
P. Andrew Evans, ${ }^{*}$ Kwong Wah Lai, and James R. Sawyer \\ Department of Chemistry, Indiana University, Bloomington, IN 47405.
}

\section{Supplemental Data}

Methyl (7aS)-2-[(4-methylphenyl)sulfonyl]-6-phenyl-2,3,7,7a-tetrahydro-1 $H$-isoindole5-carboxylate $(S)-2 a$.

$[\alpha]_{\mathrm{D}}^{25}-87.6\left(c=1.80, \mathrm{CHCl}_{3}\right)$; Chiral HPLC analysis $(25 \mathrm{~cm} \mathrm{x} 4.6$
$\mathrm{mm}$ Daicel $\mathrm{AD}-\mathrm{H}$ column, 10:90 2-propanol-hexane; $1.0 \mathrm{~mL} / \mathrm{min}$
flow rate, $240 \mathrm{~nm}$ detection light), $t_{\mathrm{R}}$ (major) $78.21 \mathrm{~min}, t_{\mathrm{R}}(\mathrm{minor})$ 1435 (s), 1346 (s), 1263 (s), 1095 (s), 1029 (s) cm ${ }^{-1} ;{ }^{1} \mathrm{H}$ NMR (400 MHz, $\left.\mathrm{CDCl}_{3}\right) \delta 7.70$ (d, J $=8.2 \mathrm{~Hz}, 2 \mathrm{H}), 7.33-7.24(\mathrm{~m}, 5 \mathrm{H}), 7.11-7.09(\mathrm{~m}, 2 \mathrm{H}), 6.16(\mathrm{~d}, J=2.0 \mathrm{~Hz}, 1 \mathrm{H}), 4.20(\mathrm{~d}, \mathrm{~A}$ of $\left.\mathrm{AB}, J_{A B}=15.8 \mathrm{~Hz}, 1 \mathrm{H}\right), 3.92(\mathrm{t}, J=8.6 \mathrm{~Hz}, 1 \mathrm{H}), 3.85\left(\mathrm{~d}, \mathrm{~B}\right.$ of AB, $\left.J_{A B}=15.4 \mathrm{~Hz}, 1 \mathrm{H}\right)$, $3.44(\mathrm{~s}, 3 \mathrm{H}), 3.08-2.96(\mathrm{~m}, 1 \mathrm{H}), 2.73(\mathrm{t}, J=9.7 \mathrm{~Hz}, 1 \mathrm{H}), 2.63(\mathrm{dd}, J=17.2,8.0 \mathrm{~Hz}, 1 \mathrm{H})$, $2.41(\mathrm{~s}, 3 \mathrm{H}), 2.25$ (t, $J=17.8 \mathrm{~Hz}, 1 \mathrm{H}) ;{ }^{13} \mathrm{C} \mathrm{NMR}\left(100 \mathrm{MHz}, \mathrm{CDCl}_{3}\right) \delta 168.00(\mathrm{e}), 143.78(\mathrm{e})$, 143.15 (e), 140.82 (e), 137.97 (e), 132.77 (e), 129.74 (o), 128.01 (o), 127.80 (o), 127.64 (o), 127.18 (o), 126.09 (e), 116.00 (o), 54.38 (e), 51.44 (o), 50.38 (e), 37.76 (o), 34.70 (e), 21.47 (o); HRMS (CI, $\mathrm{M}+\mathrm{H}^{+}$) calcd for $\mathrm{C}_{23} \mathrm{H}_{24} \mathrm{O}_{4} \mathrm{~N}_{1} \mathrm{~S}_{1} 410.1421$, found 410.1418.

\section{Methyl (7aS)-6-(4-methoxyphenyl)-2-[(4-methylphenyl)sulfonyl]-2,3,7,7a-tetrahydro-} $1 H$-isoindole-5-carboxylate $(S)-2 b$.

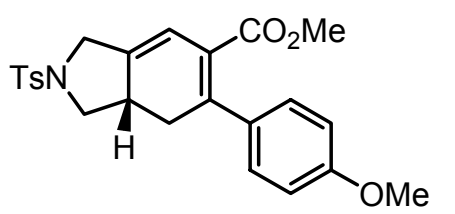

$[\alpha]_{\mathrm{D}}^{25}-77.7\left(c=0.84, \mathrm{CHCl}_{3}\right)$; Chiral HPLC analysis $(25 \mathrm{~cm} \mathrm{x}$ 4.6 mm Daicel AD-H column, 0.7:99.3 2-propanol-hexane; 0.6 $\mathrm{mL} / \mathrm{min}$ flow rate, $240 \mathrm{~nm}$ detection light), $t_{\mathrm{R}}$ (major) 293.80 min, $t_{\mathrm{R}}$ (minor) $113.30 \mathrm{~min}, 97 \%$ ee; IR (film) $2950(\mathrm{~m}), 2255$

(w), 1709 (s), 1607 (s), 1510 (s), 1347 (s), 1252 (s), 1163 (s), 1030 (s) cm ${ }^{-1}$; ${ }^{1} \mathrm{H}$ NMR (400 $\left.\mathrm{MHz}, \mathrm{CDCl}_{3}\right) \delta 7.70(\mathrm{~d}, J=8.2 \mathrm{~Hz}, 2 \mathrm{H}), 7.31(\mathrm{~d}, J=8.2 \mathrm{~Hz}, 2 \mathrm{H}), 7.06(\mathrm{~d}, J=8.6 \mathrm{~Hz}, 2 \mathrm{H})$, $6.81(\mathrm{~d}, J=8.8 \mathrm{~Hz}, 2 \mathrm{H}), 6.14(\mathrm{~d}, J=2.3 \mathrm{~Hz}, 1 \mathrm{H}), 4.19\left(\mathrm{~d}, \mathrm{~A}\right.$ of AB, $\left.J_{A B}=15.4 \mathrm{~Hz}, 1 \mathrm{H}\right)$, $3.92(\mathrm{t}, J=8.7 \mathrm{~Hz}, 1 \mathrm{H}), 3.84\left(\mathrm{~d}, \mathrm{~B}\right.$ of AB, $\left.J_{A B}=15.6 \mathrm{~Hz}, 1 \mathrm{H}\right), 3.77(\mathrm{~s}, 3 \mathrm{H}), 3.48(\mathrm{~s}, 3 \mathrm{H})$, 3.04-2.93 (m, $1 \mathrm{H}), 2.72$ (t, $J=9.7 \mathrm{~Hz}, 1 \mathrm{H}), 2.63$ (dd, $J=17.1,7.9 \mathrm{~Hz}, 1 \mathrm{H}), 2.41(\mathrm{~s}, 3 \mathrm{H})$, $2.20(\mathrm{t}, J=17.7 \mathrm{~Hz}, 1 \mathrm{H}) ;{ }^{13} \mathrm{C} \mathrm{NMR}\left(100 \mathrm{MHz}, \mathrm{CDCl}_{3}\right) \delta 168.39(\mathrm{e}), 159.36(\mathrm{e}), 143.74(\mathrm{e})$, 142.65 (e), 137.40 (e), 132.81 (e), 132.77 (e), 129.72 (o), 128.74 (o), 127.63 (o), 125.40 (e), 
116.19 (o), 113.42 (o), 55.13 (o), 54.40 (e), 51.46 (o), 50.37 (e), 37.77 (o), 34.69 (e), 21.45 (o); HRMS (CI, $\mathrm{M}^{+}$) calcd for $\mathrm{C}_{24} \mathrm{H}_{25} \mathrm{O}_{5} \mathrm{~N}_{1} \mathrm{~S}_{1} 439.1448$, found 439.1451 .

Methyl (7aS)-6-(4-methylphenyl)-2-[(4-methylphenyl)sulfonyl]-2,3,7,7a-tetrahydro-1 Hisoindole-5-carboxylate $(S)-2 c$.

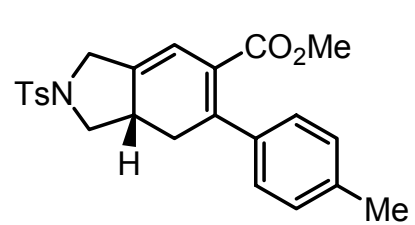

$[\alpha]_{\mathrm{D}}^{25}-87.6\left(c=1.85, \mathrm{CHCl}_{3}\right)$; Chiral HPLC analysis $(25 \mathrm{~cm} \mathrm{x}$ 4.6 mm Daicel AD-H column, 10:90 2-propanol-hexane; 1.0 $\mathrm{mL} / \mathrm{min}$ flow rate, $240 \mathrm{~nm}$ detection light), $t_{\mathrm{R}}$ (major) $115.44 \mathrm{~min}$, $t_{\mathrm{R}}$ (minor) $65.32 \mathrm{~min}, 97 \%$ ee; IR (film) 2949 (s), 2255 (m), 1713

(s), 1598 (s), 1347 (s), 1305 (s), 1164 (s) cm ${ }^{-1} ;{ }^{1} \mathrm{H}$ NMR (400 MHz, $\mathrm{CDCl}_{3}$ ) $\delta 7.71$ (d, J=8.0 $\mathrm{Hz}, 2 \mathrm{H}), 7.32$ (d, $J=8.0 \mathrm{~Hz}, 2 \mathrm{H}), 7.10$ (d, $J=7.8 \mathrm{~Hz}, 2 \mathrm{H}), 7.01$ (d, $J=8.0 \mathrm{~Hz}, 2 \mathrm{H}), 6.15$ $(\mathrm{s}, 1 \mathrm{H}), 4.20\left(\mathrm{~d}, \mathrm{~A}\right.$ of AB, $\left.J_{A B}=15.6 \mathrm{~Hz}, 1 \mathrm{H}\right), 3.93(\mathrm{t}, J=8.6 \mathrm{~Hz}, 1 \mathrm{H}), 3.85\left(\mathrm{~d}, \mathrm{~B}\right.$ of AB, $J_{A B}$ $=15.2 \mathrm{~Hz}, 1 \mathrm{H}), 3.48(\mathrm{~s}, 3 \mathrm{H}), 3.04-2.97(\mathrm{~m}, 1 \mathrm{H}), 2.73(\mathrm{t}, J=9.7 \mathrm{~Hz}, 1 \mathrm{H}), 2.63$ (dd, $J=$ 17.1, $7.9 \mathrm{~Hz}, 1 \mathrm{H}), 2.42$ (s, $3 \mathrm{H}), 2.31(\mathrm{~s}, 3 \mathrm{H}), 2.22(\mathrm{t}, J=17.9 \mathrm{~Hz}, 1 \mathrm{H}) ;{ }^{13} \mathrm{C} \mathrm{NMR}(100$ $\mathrm{MHz}, \mathrm{CDCl}_{3}$ ) $\delta 168.19$ (e), $143.76(\mathrm{e}), 143.15$ (e), 137.76 (e), 137.73 (e), 137.67 (e), 132.78 (e), 129.73 (o), 128.74 (o), 127.64 (o), 127.20 (o), 125.72 (e), 116.10 (o), 54.42 (e), 51.47 (o), 50.38 (e), 37.76 (o), 34.71 (e), 21.47 (o), 21.14 (o); HRMS (CI, M+H ${ }^{+}$) calcd for $\mathrm{C}_{24} \mathrm{H}_{26} \mathrm{O}_{4} \mathrm{~N}_{1} \mathrm{~S}_{1} 424.1577$, found 424.1576 .

Methyl (7aS)-6-(4-fluorophenyl)-2-[(4-methylphenyl)sulfonyl]-2,3,7,7a-tetrahydro-1Hisoindole-5-carboxylate $(S)-2 \mathrm{~d}$.

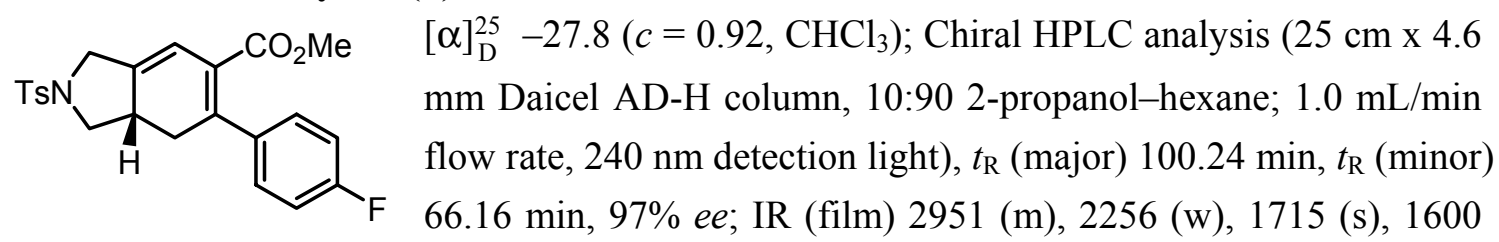
(s), 1506 (s), 1347 (s), 1227 (s), 1163 (s), 1095 (s) cm ${ }^{-1} ;{ }^{1} \mathrm{H}$ NMR (400 MHz, CDCl $)_{3} \delta$ 7.10-7.06 (m, 2 H), 6.99-6.94 (m, 2 H), 7.08 (dd, $J=8.6,5.3$ Hz, 2 H), 6.97 (t, $J=8.6$ Hz, 2 $\mathrm{H}), 6.15(\mathrm{~d}, J=2.1 \mathrm{~Hz}, 1 \mathrm{H}), 4.19\left(\mathrm{~d}, \mathrm{~A}\right.$ of $\left.\mathrm{AB}, J_{A B}=15.6 \mathrm{~Hz}, 1 \mathrm{H}\right), 3.92(\mathrm{t}, J=8.7 \mathrm{~Hz}, 1 \mathrm{H})$, $3.84\left(\mathrm{~d}, \mathrm{~B}\right.$ of $\left.\mathrm{AB}, J_{A B}=15.4 \mathrm{~Hz}, 1 \mathrm{H}\right), 3.47(\mathrm{~s}, 3 \mathrm{H}), 3.04-2.97(\mathrm{~m}, 1 \mathrm{H}), 2.72(\mathrm{t}, J=9.7 \mathrm{~Hz}, 1$ $\mathrm{H}), 2.58(\mathrm{dd}, J=17.2,8.0 \mathrm{~Hz}, 1 \mathrm{H}), 2.41(\mathrm{~s}, 3 \mathrm{H}), 2.24(\mathrm{t}, J=17.8 \mathrm{~Hz}, 1 \mathrm{H}) ;{ }^{13} \mathrm{C} \mathrm{NMR}(100$ $\left.\mathrm{MHz}, \mathrm{CDCl}_{3}\right) \delta 167.71(\mathrm{e}), 162.23\left(\mathrm{e} ; \mathrm{d}, J_{\mathrm{C}-\mathrm{F}}=247.2 \mathrm{~Hz}\right.$ ), $143.80(\mathrm{e}), 142.16(\mathrm{e}), 138.14(\mathrm{e})$, $136.78\left(\mathrm{e} ; \mathrm{d}, J_{\mathrm{C}-\mathrm{F}}=3.1 \mathrm{~Hz}\right), 132.71(\mathrm{e}), 129.74$ (o), 129.02 (o; d, $J_{\mathrm{C}-\mathrm{F}}=7.7 \mathrm{~Hz}$ ), 127.63 (o), 126.27 (e), 115.93 (o), 115.03 (o; d, $J_{\mathrm{C}-\mathrm{F}}=22.3 \mathrm{~Hz}$ ), 54.33 (e), 51.50 (o), 50.35 (e), 37.70 (o), 34.85 (e), 21.46 (o); HRMS (CI, $\mathrm{M}+\mathrm{H}^{+}$) calcd for $\mathrm{C}_{23} \mathrm{H}_{23} \mathrm{O}_{4} \mathrm{~N}_{1} \mathrm{~F}_{1} \mathrm{~S}_{1}$ 428.1326, found 428.1326. 
Methyl (7aS)-2-[(4-methylphenyl)sulfonyl]-6-[4-(trifluoromethyl)phenyl]-2,3,7,7a-tetrahydro-1 $H$-isoindole-5-carboxylate $(S)$-2e.

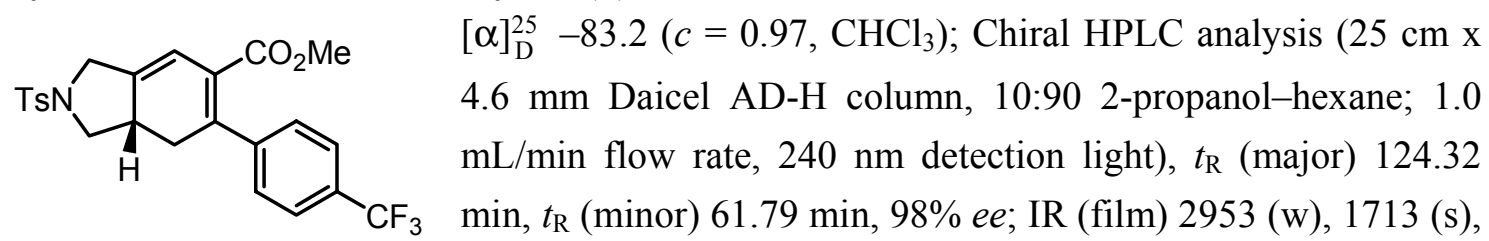
1616 (m), 1325 (s), 1163 (s), 1124 (s), 1069 (s) cm ${ }^{-1}$; ${ }^{1} \mathrm{H}$ NMR (400 MHz, $\mathrm{CDCl}_{3}$ ) $\delta 7.71$ (d, $J=8.2 \mathrm{~Hz}, 2 \mathrm{H}), 7.55(\mathrm{~d}, J=8.2 \mathrm{~Hz}, 2 \mathrm{H}), 7.33(\mathrm{~d}, J=8.2 \mathrm{~Hz}, 2 \mathrm{H}), 7.22(\mathrm{~d}, J=8.2 \mathrm{~Hz}, 2 \mathrm{H})$, $6.20(\mathrm{~d}, J=2.1 \mathrm{~Hz}, 1 \mathrm{H}), 4.22\left(\mathrm{~d}, \mathrm{~A}\right.$ of AB, $\left.J_{A B}=15.8 \mathrm{~Hz}, 1 \mathrm{H}\right), 3.93(\mathrm{t}, J=8.7 \mathrm{~Hz}, 1 \mathrm{H}), 3.86$ $\left(\mathrm{d}, \mathrm{B}\right.$ of $\left.\mathrm{AB}, J_{A B}=15.4 \mathrm{~Hz}, 1 \mathrm{H}\right), 3.47(\mathrm{~s}, 3 \mathrm{H}), 3.08-2.99(\mathrm{~m}, 1 \mathrm{H}), 2.73(\mathrm{t}, J=9.7 \mathrm{~Hz}, 1 \mathrm{H})$, 2.58 (dd, $J=17.0,8.0 \mathrm{~Hz}, 1 \mathrm{H}), 2.42(\mathrm{~s}, 3 \mathrm{H}), 2.30(\mathrm{t}, J=17.7 \mathrm{~Hz}, 1 \mathrm{H}) ;{ }^{13} \mathrm{C}$ NMR $(100 \mathrm{MHz}$, $\left.\mathrm{CDCl}_{3}\right) \delta 167.12$ (e), 144.80 (e), 143.90 (e), 141.97 (e), 139.03 (e), 132.76 (e), 129.81 (o), 129.35 (e; q, $J_{\mathrm{C}-\mathrm{F}}=33.0 \mathrm{~Hz}$ ), 127.70 (o), 127.56 (o), 126.97 (e), 125.05 (o; q, $J_{\mathrm{C}-\mathrm{F}}=3.8 \mathrm{~Hz}$ ), 123.97 (e; q, $\left.J_{\mathrm{C}-\mathrm{F}}=272.0 \mathrm{~Hz}\right), 115.80$ (o), 54.31 (e), 51.64 (o), 50.41 (e), 37.69 (o), 34.76 (e), 21.508 (o);HRMS (CI, M+H ${ }^{+}$) calcd for $\mathrm{C}_{24} \mathrm{H}_{23} \mathrm{O}_{4} \mathrm{~N}_{1} \mathrm{~F}_{3} \mathrm{~S}_{1} 478.1294$, found 478.1295.

Trimethyl (7a $R$ )-6-phenyl-1,3,7,7a-tetrahydro-2H-indene-2,2,5-tricarboxylate $(R)-2 \mathrm{f}$.

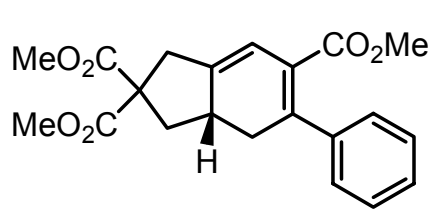

$[\alpha]_{\mathrm{D}}^{25}-23.6\left(c=0.83, \mathrm{CHCl}_{3}\right)$; Chiral HPLC analysis $(25 \mathrm{~cm} \mathrm{x}$ 4.6 mm Daicel AD-H column, 0.7:99.3 2-propanol-hexane; 0.7 $\mathrm{mL} / \mathrm{min}$ flow rate, $240 \mathrm{~nm}$ detection light), $t_{\mathrm{R}}$ (major) 124.87 $\min , t_{\mathrm{R}}$ (minor) $134.75 \mathrm{~min}, \geq 99 \%$ ee; IR (neat) $2953(\mathrm{~m}), 1733$ (s), 1435 (s), 1251 (s), 1201 (s), 1169 (m), 1068 (m) cm ${ }^{-1} ;{ }^{1} \mathrm{H}$ NMR (400 MHz, CDCl $) \delta$ 7.31-7.22 (m, $3 \mathrm{H}), 7.16-7.14$ (m, $2 \mathrm{H}), 6.18$ (d, $J=2.3 \mathrm{~Hz}, 1 \mathrm{H}), 3.73$ (s, $3 \mathrm{H}), 3.72$ (s, $3 \mathrm{H})$, $3.46(\mathrm{~s}, 3 \mathrm{H}), 3.24\left(\mathrm{~d}, \mathrm{~A}\right.$ of AB, $\left.J_{A B}=18.6 \mathrm{~Hz}, 1 \mathrm{H}\right), 3.06\left(\mathrm{~d}, \mathrm{~B}\right.$ of AB, $\left.J_{A B}=18.6 \mathrm{~Hz}, 1 \mathrm{H}\right)$, 2.89-2.82 (m, $1 \mathrm{H}), 2.71(\mathrm{t}, J=7.8 \mathrm{~Hz}, 1 \mathrm{H}), 2.68(\mathrm{dd}, J=11.7,7.4 \mathrm{~Hz}, 1 \mathrm{H}), 2.33$ (t, $J=$ $17.7 \mathrm{~Hz}, 1 \mathrm{H}), 1.89$ (t, $J=11.9 \mathrm{~Hz}, 1 \mathrm{H}) ;{ }^{13} \mathrm{C} \mathrm{NMR}\left(100 \mathrm{MHz}, \mathrm{CDCl}_{3}\right) \delta 171.94$ (e), 171.82 (e), 168.65 (e), 143.14 (e), 142.73 (e), 141.53 (e), 127.98 (o), 127.49 (o), 127.33 (o), 126.80 (e), 116.22 (o), 59.42 (e), 52.87 (o), 51.38 (o), 40.49 (e), 38.50 (o), 38.10 (e), 37.92 (o), 37.01 (e); HRMS (CI, $\mathrm{M}^{+}$) calcd for $\mathrm{C}_{21} \mathrm{H}_{22} \mathrm{O}_{6} 370.1411$, found 370.1397 .

Trimethyl (7aR)-6-(4-methoxyphenyl)-1,3,7,7a-tetrahydro- $2 H$-indene-2,2,5-tricarboxylate $(R)-2 g$.

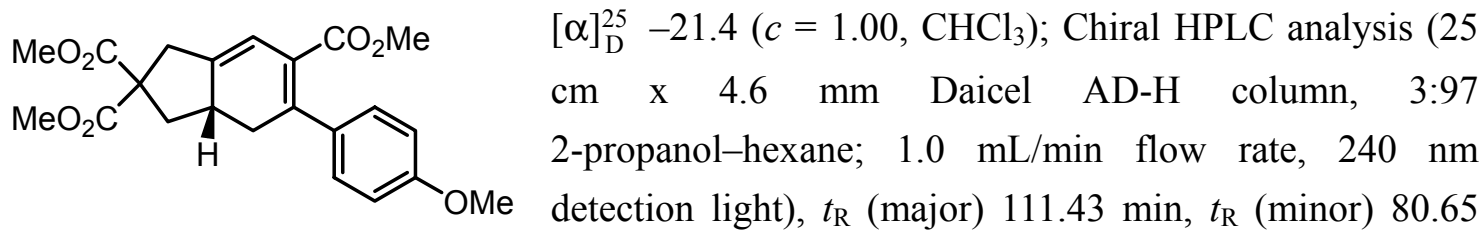

min, 98\% ee; IR (neat) 2953 (m), 1733 (s), 1607 (m), 1510 (s), 1435 (s), 1250 (s), 1176 (s) $\mathrm{cm}^{-1} ; \quad{ }^{1} \mathrm{H}$ NMR $\left(400 \mathrm{MHz}, \mathrm{CDCl}_{3}\right) \delta 7.08(\mathrm{~d}, J=8.6 \mathrm{~Hz}, 2 \mathrm{H}), 6.82(\mathrm{~d}, J=8.6 \mathrm{~Hz}, 2 \mathrm{H})$, 
$6.15(\mathrm{~d}, J=2.3 \mathrm{~Hz}, 1 \mathrm{H}), 3.77(\mathrm{~s}, 3 \mathrm{H}), 3.72(\mathrm{~s}, 3 \mathrm{H}), 3.71(\mathrm{~s}, 3 \mathrm{H}), 3.49$ (s, $3 \mathrm{H}), 3.22(\mathrm{~d}, \mathrm{~A}$ of $\left.\mathrm{AB}, J_{A B}=18.8 \mathrm{~Hz}, 1 \mathrm{H}\right), 3.04\left(\mathrm{~d}, \mathrm{~B}\right.$ of AB, $\left.J_{A B}=18.4 \mathrm{~Hz}, 1 \mathrm{H}\right), 2.85-2.77(\mathrm{~m}, 1 \mathrm{H}), 2.71(\mathrm{t}, J$ $=6.6 \mathrm{~Hz}, 1 \mathrm{H}), 2.67(\mathrm{dd}, J=10.1,7.1 \mathrm{~Hz}, 1 \mathrm{H}), 2.27$ (t, $J=17.6 \mathrm{~Hz}, 1 \mathrm{H}), 1.88(\mathrm{dd}, J=12.5$, $11.1 \mathrm{~Hz}, 1 \mathrm{H}) ;{ }^{13} \mathrm{C}$ NMR (100 MHz, $\left.\mathrm{CDCl}_{3}\right) \delta 171.89$ (e), 171.78 (e), 168.96 (e), 159.10 (e), 142.60 (e), 142.15 (e), 133.54 (e), 128.78 (o), 126.11 (e), 116.34 (o), 113.36 (o), 59.40 (e), 55.12 (o), 52.79 (o), 51.34 (o), 40.44 (e), 38.45 (o), 38.03 (e), 37.89 (o), 36.96 (e); HRMS (CI, $\mathrm{M}^{+}$) calcd for $\mathrm{C}_{22} \mathrm{H}_{24} \mathrm{O}_{7} 400.1517$, found 400.1514 .

Trimethyl (7a $R$ )-6-(4-methylphenyl)-1,3,7,7a-tetrahydro-2H-indene-2,2,5-tricarboxylate $(R)-2 h$.

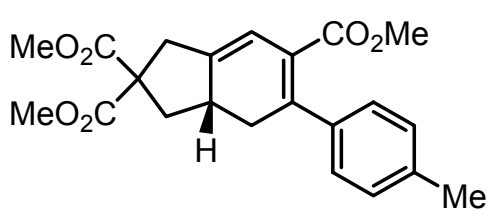
$[\alpha]_{\mathrm{D}}^{25}-25.1\left(c=0.94, \mathrm{CHCl}_{3}\right)$; Chiral HPLC analysis $(25$ $\mathrm{cm} \quad$ x $\quad 4.6 \mathrm{~mm}$ Daicel AD-H column, 0.7:99.3 2-propanol-hexane; $0.7 \mathrm{~mL} / \mathrm{min}$ flow rate, $240 \mathrm{~nm}$ detection

light), $t_{\mathrm{R}}$ (major) $210.47 \mathrm{~min}, t_{\mathrm{R}}$ (minor) $187.88 \mathrm{~min}, 95 \%$ ee; IR (neat) 2952 (m), 1733 (s), 1435 (s), 1250 (s), 1200 (s), 1169 (s), 1067 (m) cm ${ }^{-1}$; ${ }^{1} \mathrm{H}$ NMR $\left(400 \mathrm{MHz}, \mathrm{CDCl}_{3}\right) \delta$ 7.24-7.03 (m, $\left.4 \mathrm{H}\right), 6.16(\mathrm{~s}, 1 \mathrm{H}), 3.72(\mathrm{~s}, 3 \mathrm{H}), 3.71(\mathrm{~s}, 3 \mathrm{H}), 3.49(\mathrm{~s}, 3$ $\mathrm{H}), 3.23\left(\mathrm{~d}, \mathrm{~A}\right.$ of AB, $\left.J_{A B}=18.8 \mathrm{~Hz}, 1 \mathrm{H}\right), 3.05\left(\mathrm{~d}, \mathrm{~B}\right.$ of AB, $\left.J_{A B}=18.6 \mathrm{~Hz}, 1 \mathrm{H}\right), 2.87-2.80$ $(\mathrm{m}, 1 \mathrm{H}), 2.71(\mathrm{t}, J=7.8 \mathrm{~Hz}, 1 \mathrm{H}), 2.67(\mathrm{dd}, J=12.1,7.4 \mathrm{~Hz}, 1 \mathrm{H}), 2.31(\mathrm{~s}, 3 \mathrm{H}), 2.29(\mathrm{t}, J=$ $18.0 \mathrm{~Hz}, 1 \mathrm{H}), 1.89$ (t, $J=11.8 \mathrm{~Hz}, 1 \mathrm{H}) ;{ }^{13} \mathrm{C} \mathrm{NMR}\left(100 \mathrm{MHz}, \mathrm{CDCl}_{3}\right) \delta 171.91(\mathrm{e}), 171.80$ (e), 168.78 (e), 143.12 (e), 142.41 (e), 138.41 (e), 137.31 (e), 128.68 (o), 127.30 (o), 126.41 (e), 116.28 (e), 59.41 (e), 52.82 (o), 51.36 (o), 40.48 (e), 38.47 (o), 38.06 (e), 37.89 (o), 37.00 (e), 21.16 (o); HRMS (CI, $\mathrm{M}^{+}$) calcd for $\mathrm{C}_{22} \mathrm{H}_{25} \mathrm{O}_{6} 384.1567$, found 384.1579.

\section{Trimethyl (7a $R$ )-6-(4-fluorophenyl)-1,3,7,7a-tetrahydro-2H-indene-2,2,5-tricarboxy-} late $(R)-2 i$.

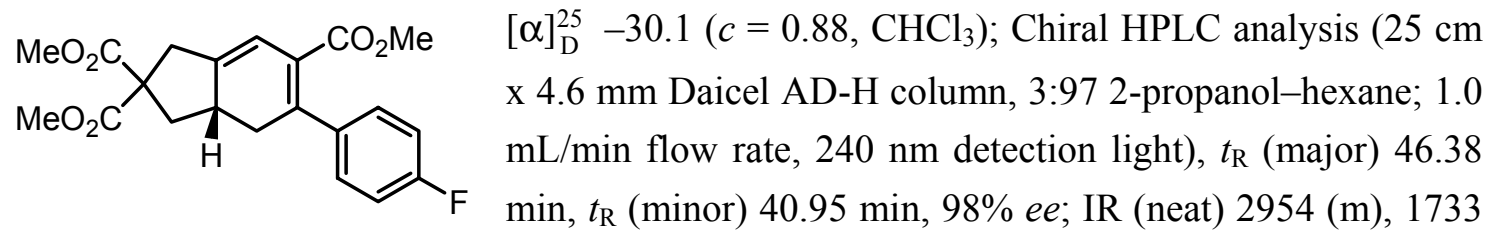
(s), 1602 (mw), 1507 (m), 1435 (m), 1250 (s), 1202 (m), 1161 (m), 1068 (m) cm ${ }^{-1}$; ${ }^{1} \mathrm{H}$ NMR (400 MHz, $\left.\mathrm{CDCl}_{3}\right) \delta 7.14-7.09$ (m, $\left.2 \mathrm{H}\right), 7.00-6.96(\mathrm{~m}, 2 \mathrm{H}), 6.17(\mathrm{~d}, J=2.1 \mathrm{~Hz}, 1 \mathrm{H}), 3.73$ $(\mathrm{s}, 3 \mathrm{H}), 3.72(\mathrm{~s}, 3 \mathrm{H}), 3.49(\mathrm{~s}, 3 \mathrm{H}), 3.24\left(\mathrm{~d}, \mathrm{~A}\right.$ of AB, $\left.J_{A B}=18.6 \mathrm{~Hz}, 1 \mathrm{H}\right), 3.05(\mathrm{~d}, \mathrm{~B}$ of AB, $\left.J_{A B}=18.6 \mathrm{~Hz}, 1 \mathrm{H}\right), 2.88-2.81(\mathrm{~m}, 1 \mathrm{H}), 2.70(\mathrm{dd}, J=12.7,7.6 \mathrm{~Hz}, 1 \mathrm{H}), 2.63(\mathrm{dd}, J=16.8$, $7.6 \mathrm{~Hz}, 1 \mathrm{H}), 2.31(\mathrm{t}, J=17.7 \mathrm{~Hz}, 1 \mathrm{H}), 1.89(\mathrm{t}, J=11.8 \mathrm{~Hz}, 1 \mathrm{H}) ;{ }^{13} \mathrm{C}$ NMR $(100 \mathrm{MHz}$, $\left.\mathrm{CDCl}_{3}\right) \delta 171.89$ (e), 171.77 (e), 168.36 (e), 162.14 (e; d, $J_{\mathrm{C}-\mathrm{F}}=247.2 \mathrm{~Hz}$ ), 142.91 (e), 142.12 (e), 137.47 (e; d, $\left.J_{\mathrm{C}-\mathrm{F}}=3.0 \mathrm{~Hz}\right), 129.08$ (o; d, $\left.J_{\mathrm{C}-\mathrm{F}}=8.4 \mathrm{~Hz}\right), 126.97$ (e), 116.15 (o), 114.96 (o; d, $J_{\mathrm{C}-\mathrm{F}}=21.5 \mathrm{~Hz}$ ), 59.40 (e), 52.87 (o), 51.43 (o), 40.42 (e), 38.47 (o), 38.09 (e), 37.86 (o), 37.16 (e); HRMS (CI, M ${ }^{+}$) calcd for $\mathrm{C}_{21} \mathrm{H}_{21} \mathrm{O}_{6} \mathrm{~F}_{1} 388.1317$, found 388.1312. 
Trimethyl (7aR)-6-[4-(trifluoromethyl)phenyl]-1,3,7,7a-tetrahydro-2H-indene-2,2,5tricarboxylate $(R)-2 \mathbf{j}$.

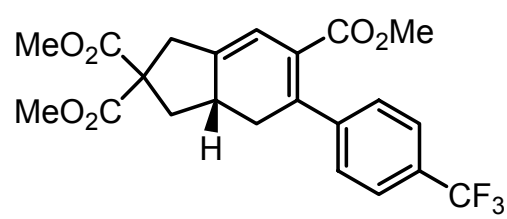
$[\alpha]_{\mathrm{D}}^{25}-39.8\left(c=1.45, \mathrm{CHCl}_{3}\right)$; Chiral HPLC analysis $(25$ $\mathrm{cm} \quad \mathrm{x} \quad 4.6 \mathrm{~mm}$ Daicel AD-H column, 0.7:99.3 2-propanol-hexane; $0.7 \mathrm{~mL} / \mathrm{min}$ flow rate, $240 \mathrm{~nm}$ detection light), $t_{\mathrm{R}}$ (major) $216.39 \mathrm{~min}, t_{\mathrm{R}}$ (minor) 167.64 min, 98\% ee; IR (neat) 2955 (m), 1733 (s), 1616 (m), 1436 (s), 1326 (s), 1252 (s), 1167 (s), 1125 (s), 1069 (s) cm ${ }^{-1} ;{ }^{1} \mathrm{H}$ NMR (400 MHz, $\mathrm{CDCl}_{3}$ ) $\delta 7.57$ (d, $\left.J=8.2 \mathrm{~Hz}, 2 \mathrm{H}\right), 7.27$ (d, $J=$ $8.6 \mathrm{~Hz}, 2 \mathrm{H}), 6.22(\mathrm{~d}, J=2.2 \mathrm{~Hz}, 1 \mathrm{H}), 3.75(\mathrm{~s}, 3 \mathrm{H}), 3.74(\mathrm{~s}, 3 \mathrm{H}), 3.50(\mathrm{~s}, 3 \mathrm{H}), 3.27(\mathrm{~d}, \mathrm{~A}$ of $\left.\mathrm{AB}, J_{A B}=18.6 \mathrm{~Hz}, 1 \mathrm{H}\right), 3.09\left(\mathrm{~d}, \mathrm{~B}\right.$ of AB, $\left.J_{A B}=18.8 \mathrm{~Hz}, 1 \mathrm{H}\right), 2.97-2.86(\mathrm{~m}, 1 \mathrm{H}), 2.73(\mathrm{dd}$, $J=12.6,7.5 \mathrm{~Hz}, 1 \mathrm{H}), 2.64(\mathrm{dd}, J=16.8,7.6 \mathrm{~Hz}, 1 \mathrm{H}), 2.38(\mathrm{t}, J=17.7 \mathrm{~Hz}, 1 \mathrm{H}), 1.92(\mathrm{t}, J=$ $11.9 \mathrm{~Hz}, 1 \mathrm{H}) ;{ }^{13} \mathrm{C} \mathrm{NMR}\left(100 \mathrm{MHz}, \mathrm{CDCl}_{3}\right) \delta 171.86$ (e), 171.71 (e), 167.75 (e), $149.82(\mathrm{e})$, 145.47 (e), 143.80 (e), 141.89 (e), 129.34 (e; q, $J_{\mathrm{C}-\mathrm{F}}=33.0 \mathrm{~Hz}$ ), 127.64 (o), 124.97 (o; q, $J_{\mathrm{C}-\mathrm{F}}$ $=3.8 \mathrm{~Hz}$ ), 124.09 (e; q, $\left.J_{\mathrm{C}-\mathrm{F}}=271.8 \mathrm{~Hz}\right), 115.99$ (o), 59.35 (e), 52.92 (o), 51.52 (o), 40.37 (e), 38.48 (o), 38.13 (e), 37.79 (o), 37.01 (e); HRMS (CI, M ${ }^{+}$) calcd for $\mathrm{C}_{22} \mathrm{H}_{21} \mathrm{O}_{6} \mathrm{~F}_{3} 438.1285$, found 438.1290 .

Methyl (7aS)-6-phenyl-1,3,7,7a-tetrahydro-2-benzofuran-5-carboxylate $(S)$-2k.

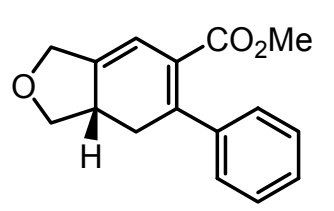
$[\alpha]_{\mathrm{D}}^{25}-13.9\left(c=2.39, \mathrm{CHCl}_{3}\right)$; Chiral HPLC analysis $(25 \mathrm{~cm} \times 4.6 \mathrm{~mm}$ Daicel AD-H column, 0.7:99.3 2-propanol-hexane; $0.6 \mathrm{~mL} / \mathrm{min}$ flow rate, $240 \mathrm{~nm}$ detection light), $t_{\mathrm{R}}$ (major) $38.43 \mathrm{~min}, t_{\mathrm{R}}$ (minor) 48.98 min, $\geq 99 \%$ ee; IR (neat) 2949 (s), 2853 (s), 1714 (s), 1584 (m), 1435 (s), 1252 (s), 1161 (s), 1028 (s) cm ${ }^{-1} ;{ }^{1} \mathrm{H}$ NMR (400 MHz, $\left.\mathrm{CDCl}_{3}\right) \delta$ 7.34-7.24 (m, $3 \mathrm{H}$ ), 7.19-7.17 (m, $2 \mathrm{H}), 6.21(\mathrm{~d}, J=2.1 \mathrm{~Hz}, 1 \mathrm{H}), 4.60$ (d, A of AB, $\left.J_{A B}=14.7 \mathrm{~Hz}, 1 \mathrm{H}\right), 4.42(\mathrm{~d}$, $\mathrm{B}$ of $\left.\mathrm{AB}, J_{A B}=14.7 \mathrm{~Hz}, 1 \mathrm{H}\right), 4.33(\mathrm{t}, J=8.0 \mathrm{~Hz}, 1 \mathrm{H}), 3.48(\mathrm{~s}, 3 \mathrm{H}), 3.43(\mathrm{dd}, J=9.8,8.4 \mathrm{~Hz}$, $1 \mathrm{H}), 3.12-3.01(\mathrm{~m}, 1 \mathrm{H}), 2.68(\mathrm{dd}, J=17.0,7.8 \mathrm{~Hz}, 1 \mathrm{H}), 2.38(\mathrm{t}, J=17.7 \mathrm{~Hz}, 1 \mathrm{H}) ;{ }^{13} \mathrm{C}$ NMR (100 MHz, $\mathrm{CDCl}_{3}$ ) $\delta 168.45$ (e), 143.07 (e), 142.75 (e), 141.37 (e), 128.02 (o), 127.64 (o), 127.32 (o), 126.87 (e), 113.37 (o), 74.45 (e), 69.22 (e), 51.42 (o), 39.29 (o), 34.03 (e); HRMS (CI, M ${ }^{+}$) calcd for $\mathrm{C}_{16} \mathrm{H}_{16} \mathrm{O}_{3} 256.1094$, found 256.1088 .

\section{Methyl (7aS)-6-(4-methoxyphenyl)-1,3,7,7a-tetrahydro-2-benzofuran-5-carboxylate} (S)-21.

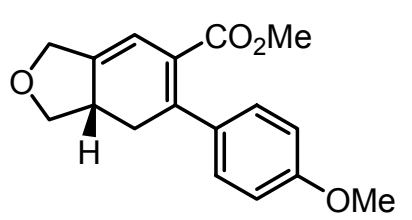

$[\alpha]_{\mathrm{D}}^{25}-15.4\left(c=4.00, \mathrm{CHCl}_{3}\right)$; Chiral HPLC analysis $(25 \mathrm{~cm} \mathrm{x}$ $4.6 \mathrm{~mm}$ Daicel AD-H column, 1:99 2-propanol-hexane; 1.0 $\mathrm{mL} / \mathrm{min}$ flow rate, $240 \mathrm{~nm}$ detection light), $t_{\mathrm{R}}$ (major) $56.62 \mathrm{~min}$, $t_{\mathrm{R}}$ (minor) $53.39 \mathrm{~min}, 98 \% \mathrm{ee}$; IR (neat) $2949(\mathrm{~m}), 1710(\mathrm{~s}), 1607$

(s), 1510 (s), 1252 (s), 1179 (s), 1027 (s) cm ${ }^{-1} ;{ }^{1} \mathrm{H}$ NMR (400 MHz, CDCl ${ }_{3}$ ) 7.14-7.12 (m, 2 H), 6.85-6.83 (m, $2 \mathrm{H}), 6.19(\mathrm{~d}, J=2.3 \mathrm{~Hz}, 1 \mathrm{H}), 4.58\left(\mathrm{~d}, \mathrm{~A}\right.$ of AB, $\left.J_{A B}=14.5 \mathrm{~Hz}, 1 \mathrm{H}\right), 4.41$ 
(d, B of AB, $\left.J_{A B}=14.5 \mathrm{~Hz}, 1 \mathrm{H}\right), 4.32(\mathrm{t}, J=8.0 \mathrm{~Hz}, 1 \mathrm{H}), 3.79(\mathrm{~s}, 3 \mathrm{H}), 3.52(\mathrm{~s}, 3 \mathrm{H}), 3.42$ $(\mathrm{dd}, J=10.1,8.9 \mathrm{~Hz}, 1 \mathrm{H}), 3.10-2.98(\mathrm{~m}, 1 \mathrm{H}), 2.68(\mathrm{dd}, J=16.9,7.7 \mathrm{~Hz}, 1 \mathrm{H}), 2.33$ (t, $J=$ $17.7 \mathrm{~Hz}, 1 \mathrm{H}) ;{ }^{13} \mathrm{C}$ NMR (100 MHz, $\left.\mathrm{CDCl}_{3}\right) \delta 168.82$ (e), 159.25 (e), 142.59 (e), 142.20 (e), 133.39 (e), 128.83 (o), 126.20 (e), 113.54 (o), 113.43 (o), 74.46 (e), 69.21 (e), 55.15 (o), 51.44 (o), 39.32 (o), 34.02 (e); HRMS (CI, $\mathrm{M}^{+}$) calcd for $\mathrm{C}_{17} \mathrm{H}_{18} \mathrm{O}_{4}$ 286.1200, found 286.1188 .

\section{Methyl (7aS)-6-(4-methylphenyl)-1,3,7,7a-tetrahydro-2-benzofuran-5-carboxylate} (S)-2m.

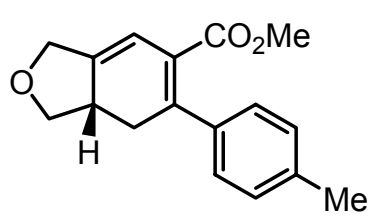
$[\alpha]_{\mathrm{D}}^{25}-28.2\left(c=1.91, \mathrm{CHCl}_{3}\right)$; Chiral HPLC analysis $(25 \mathrm{~cm} \mathrm{x} 4.6$ $\mathrm{mm}$ Daicel AD-H column, 0.8:99.2 2-propanol-hexane; 0.8 $\mathrm{mL} / \mathrm{min}$ flow rate, $240 \mathrm{~nm}$ detection light), $t_{\mathrm{R}}$ (major) $39.72 \mathrm{~min}, t_{\mathrm{R}}$ (minor) $41.75 \mathrm{~min}, 98 \%$ ee; IR (neat) $2948(\mathrm{~m}), 1712(\mathrm{~s}), 1511(\mathrm{~m})$, 1435 (m), 1249 (m), 1160 (m), 1029 (m) cm ${ }^{-1} ;{ }^{1} \mathrm{H}$ NMR (400 MHz, CDCl ${ }_{3}$ ) $\delta$ 7.14-7.08 (m, 4 $\mathrm{H}), 6.20(\mathrm{~d}, J=2.3 \mathrm{~Hz}, 1 \mathrm{H}), 4.60\left(\mathrm{~d}, \mathrm{~A}\right.$ of AB, $\left.J_{A B}=14.7 \mathrm{~Hz}, 1 \mathrm{H}\right), 4.42\left(\mathrm{~d}, \mathrm{~B}\right.$ of AB, $J_{A B}=$ $14.7 \mathrm{~Hz}, 1 \mathrm{H}), 4.33(\mathrm{t}, J=8.0 \mathrm{~Hz}, 1 \mathrm{H}), 3.52(\mathrm{~s}, 3 \mathrm{H}), 3.43(\mathrm{dd}, J=9.8,8.4 \mathrm{~Hz}, 1 \mathrm{H})$, 3.12-3.00 (m, $1 \mathrm{H}), 2.68$ (dd, $J=16.9,7.7 \mathrm{~Hz}, 1 \mathrm{H}), 2.35$ (t, $J=17.7 \mathrm{~Hz}, 1 \mathrm{H}), 2.34$ (s, $3 \mathrm{H})$; ${ }^{13} \mathrm{C}$ NMR (100 MHz, $\left.\mathrm{CDCl}_{3}\right) \delta 168.61$ (e), 143.07 (e), 142.44 (e), 138.26 (e), 137.52 (e), 128.72 (o), 127.31 (o), 126.48 (e), 113.45 (o), 74.45 (e), 69.20 (e), 51.42 (o), 39.28 (o), 34.02 (e), 21.17 (o); HRMS (CI, $\mathrm{M}^{+}$) calcd for $\mathrm{C}_{17} \mathrm{H}_{18} \mathrm{O}_{3} 270.1250$, found 270.1241 .

Methyl (7aS)-6-(4-fluorophenyl)-1,3,7,7a-tetrahydro-2-benzofuran-5-carboxylate $(S)-2 n$.

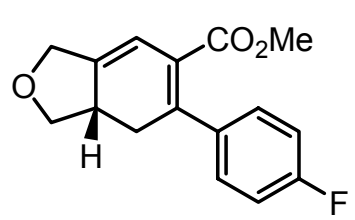
$[\alpha]_{\mathrm{D}}^{25}-29.2\left(c=2.63, \mathrm{CHCl}_{3}\right)$; Chiral HPLC analysis $(25 \mathrm{~cm} \mathrm{x} 4.6$ mm Daicel AD-H column, 0.7:99.3 2-propanol-hexane; $0.6 \mathrm{~mL} / \mathrm{min}$ flow rate, $240 \mathrm{~nm}$ detection light), $t_{\mathrm{R}}$ (major) $66.28 \mathrm{~min}, t_{\mathrm{R}}$ (minor) $96.85 \mathrm{~min}, \geq 99 \%$ ee; IR (neat) 2951 (m), 1715 (s), 1602 (m), 1507 (s), 1436 (m), 1227 (s), 1161 (s) cm ${ }^{-1} ;{ }^{1} \mathrm{H}$ NMR (400 MHz, $\left.\mathrm{CDCl}_{3}\right) \delta$ 7.17-7.13 (m, $\left.2 \mathrm{H}\right)$, $7.02-6.98(\mathrm{~m}, 2 \mathrm{H}), 6.20(\mathrm{~d}, J=2.3 \mathrm{~Hz}, 1 \mathrm{H}), 4.59$ (d, A of AB, $\left.J_{A B}=14.3 \mathrm{~Hz}, 1 \mathrm{H}\right), 4.42(\mathrm{~d}$, B of $\left.\mathrm{AB}, J_{A B}=14.7 \mathrm{~Hz}, 1 \mathrm{H}\right), 4.32(\mathrm{t}, J=8.0 \mathrm{~Hz}, 1 \mathrm{H}), 3.51(\mathrm{~s}, 3 \mathrm{H}), 3.42(\mathrm{dd}, J=9.8,8.4 \mathrm{~Hz}$, $1 \mathrm{H}), 3.11-3.00(\mathrm{~m}, 1 \mathrm{H}), 2.63(\mathrm{dd}, J=16.9,7.7 \mathrm{~Hz}, 1 \mathrm{H}), 2.37(\mathrm{t}, J=17.7 \mathrm{~Hz}, 1 \mathrm{H}) ;{ }^{13} \mathrm{C}$ NMR (100 MHz, $\left.\mathrm{CDCl}_{3}\right) \delta 168.17(\mathrm{e}), 162.21\left(\mathrm{e} ; \mathrm{d}, J_{\mathrm{C}-\mathrm{F}}=247.2 \mathrm{~Hz}\right), 142.92(\mathrm{e}), 142.07$ (e), $137.31\left(\mathrm{e} ; \mathrm{d}, J_{\mathrm{C}-\mathrm{F}}=3.1 \mathrm{~Hz}\right), 129.11\left(\mathrm{o} ; \mathrm{d}, J_{\mathrm{C}-\mathrm{F}}=8.4 \mathrm{~Hz}\right), 127.05(\mathrm{e}), 115.02\left(\mathrm{o} ; \mathrm{d}, J_{\mathrm{C}-\mathrm{F}}=21.5\right.$ Hz), 113.30 (o), 74.38 (e), 69.20 (e), 51.48 (o), 39.25 (o), 34.18 (e); HRMS (CI, M ${ }^{+}$) calcd for $\mathrm{C}_{16} \mathrm{H}_{15} \mathrm{O}_{3} \mathrm{~F}_{1} 274.1000$, found 274.0991. 
Methyl (7aS)-6-[4-(trifluoromethyl)phenyl]-1,3,7,7a-tetrahydro-2-benzofuran-5-carboxylate $(S)-20$.

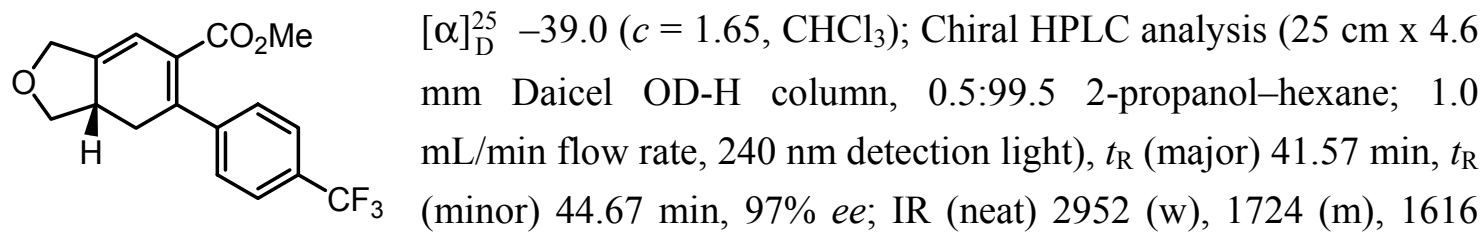

(w), 1326 (s), 1164 (s), 1124 (s), 1069 (s) cm ${ }^{-1} ;{ }^{1} \mathrm{H}$ NMR (400 MHz, $\mathrm{CDCl}_{3}$ ) $\delta 7.57$ (d, $J=$ $8.2 \mathrm{~Hz}, 2 \mathrm{H}), 7.29(\mathrm{~d}, J=8.2 \mathrm{~Hz}, 2 \mathrm{H}), 6.24(\mathrm{~d}, J=2.3 \mathrm{~Hz}, 1 \mathrm{H}), 4.61\left(\mathrm{~d}, \mathrm{~A}\right.$ of AB, $J_{A B}=14.8$ $\mathrm{Hz}, 1 \mathrm{H}), 4.43\left(\mathrm{~d}, \mathrm{~B}\right.$ of AB, $\left.J_{A B}=14.8 \mathrm{~Hz}, 1 \mathrm{H}\right), 4.34(\mathrm{t}, J=8.0 \mathrm{~Hz}, 1 \mathrm{H}), 3.51(\mathrm{~s}, 3 \mathrm{H}), 3.44$ $(\mathrm{dd}, J=9.7,8.5 \mathrm{~Hz}, 1 \mathrm{H}), 3.14-3.03(\mathrm{~m}, 1 \mathrm{H}), 2.62(\mathrm{dd}, J=17.0,7.8 \mathrm{~Hz}, 1 \mathrm{H}), 2.42(\mathrm{t}, J=$ $17.7 \mathrm{~Hz}, 1 \mathrm{H}) ;{ }^{13} \mathrm{C}$ NMR (100 MHz, $\left.\mathrm{CDCl}_{3}\right) \delta 167.59$ (e), 145.34 (e), 143.79 (e), 141.88 (e), $129.54\left(\mathrm{e} ; \mathrm{q}, J_{\mathrm{C}-\mathrm{F}}=32.5 \mathrm{~Hz}\right), 127.75(\mathrm{e}), 127.67$ (o), 125.04 (o; q, $\left.J_{\mathrm{C}-\mathrm{F}}=3.8 \mathrm{~Hz}\right), 124.06$ (e; q, $J_{\mathrm{C}-\mathrm{F}}=272.0 \mathrm{~Hz}$ ), 113.18 (o), 74.37 (e), 69.24 (e), 51.61 (o), 39.20 (o), 34.07 (e); HRMS (CI, $\mathrm{M}^{+}$) calcd for $\mathrm{C}_{17} \mathrm{H}_{15} \mathrm{O}_{3} \mathrm{~F}_{3} 324.0968$, found 324.0965.

\section{1-\{(7aS)-2-[(4-Methylphenyl)sulfonyl]-6-phenyl-2,3,7,7a-tetrahydro-1 $H$-isoindol-5-yl\}- ethanone $(S)-4 a$.}

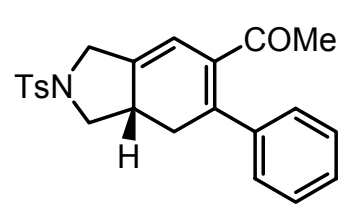

$[\alpha]_{\mathrm{D}}^{25}-56.8\left(c=1.70, \mathrm{CHCl}_{3}\right)$; Chiral HPLC analysis $(25 \mathrm{~cm} \times 4.6$ $\mathrm{mm}$ Daicel AD-H column, 4:96 2-propanol-hexane; $1.0 \mathrm{~mL} / \mathrm{min}$ flow rate, $240 \mathrm{~nm}$ detection light), $t_{\mathrm{R}}$ (major) $190.62 \mathrm{~min}, t_{\mathrm{R}}$ (minor) 112.24 min, 95\% ee; IR (film) 2862 (m), 2254 (w),1668 (s), 1347 (s), 1166 (s), 1095 (s); ${ }^{1} \mathrm{H}$ NMR (400 MHz, $\left.\mathrm{CDCl}_{3}\right) \delta 7.70$ (d, $\left.J=8.0 \mathrm{~Hz}, 2 \mathrm{H}\right), 7.32-7.31$ (m, $\left.5 \mathrm{H}\right)$, 7.14-7.13 (m, $2 \mathrm{H}), 6.13(\mathrm{~s}, 1 \mathrm{H}), 4.18\left(\mathrm{~d}, \mathrm{~A}\right.$ of AB, $\left.J_{A B}=15.4 \mathrm{~Hz}, 1 \mathrm{H}\right), 3.94(\mathrm{t}, J=8.6 \mathrm{~Hz}, 1$ $\mathrm{H}), 3.82\left(\mathrm{~d}, \mathrm{~B}\right.$ of AB, $\left.J_{A B}=15.4 \mathrm{~Hz}, 1 \mathrm{H}\right), 3.07-2.98(\mathrm{~m}, 1 \mathrm{H}), 2.71(\mathrm{t}, J=9.7 \mathrm{~Hz}, 1 \mathrm{H}), 2.66$ $(\mathrm{dd}, J=17.3,7.9 \mathrm{~Hz}, 1 \mathrm{H}), 2.41(\mathrm{~s}, 3 \mathrm{H}), 2.22(\mathrm{t}, J=17.8 \mathrm{~Hz}, 1 \mathrm{H}), 1.69(\mathrm{~s}, 3 \mathrm{H}) ;{ }^{13} \mathrm{C} \mathrm{NMR}$ $\left(100 \mathrm{MHz}, \mathrm{CDCl}_{3}\right) \delta 203.28$ (e), 143.76 (e), 141.15 (e), 140.35 (e), 137.77 (e), 135.77 (e), 132.70 (e), 129.73 (o), 128.68 (o), 128.62 (o), 128.10 (o), 127.66 (o), 116.13 (o), 54.42 (e), 50.38 (e), 37.88 (o), 34.51 (e), 30.13 (o), 21.47 (o); HRMS (CI, M+H ${ }^{+}$) calcd for $\mathrm{C}_{23} \mathrm{H}_{24} \mathrm{O}_{3} \mathrm{~N}_{1} \mathrm{~S}_{1}$ 394.1471, found 394.1470.

1-\{(7aS)-7a-Methyl-2-[(4-methylphenyl)sulfonyl]-6-phenyl-2,3,7,7a-tetrahydro- $1 \mathrm{H}$-isoindol-5-yl\}ethanone $(S)-4 a^{\prime}$.

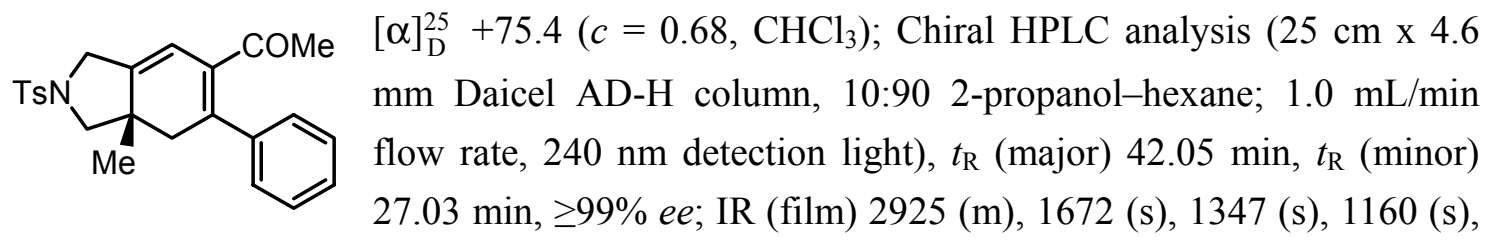

1093 (s); ${ }^{1} \mathrm{H}$ NMR (400 MHz, $\left.\mathrm{CDCl}_{3}\right) \delta 7.70$ (d, $\left.J=8.2 \mathrm{~Hz}, 2 \mathrm{H}\right), 7.34-7.31$ (m, $\left.5 \mathrm{H}\right)$, 7.13-7.11 (m, $2 \mathrm{H}), 6.06(\mathrm{~s}, 1 \mathrm{H}), 4.24\left(\mathrm{~d}, \mathrm{~A}\right.$ of AB, $\left.J_{A B}=15.3 \mathrm{~Hz}, 1 \mathrm{H}\right), 3.82\left(\mathrm{~d}, \mathrm{~B}\right.$ of AB, $J_{A B}$ 
$=15.4 \mathrm{~Hz}, 1 \mathrm{H}), 3.58\left(\mathrm{~d}, \mathrm{~A}\right.$ of AB, $\left.J_{A B}=9.0 \mathrm{~Hz}, 1 \mathrm{H}\right), 2.81\left(\mathrm{~d}, \mathrm{~B}\right.$ of AB, $\left.J_{A B}=9.2 \mathrm{~Hz}, 1 \mathrm{H}\right)$, $2.50(\mathrm{~s}, 2 \mathrm{H}), 2.42(\mathrm{~s}, 3 \mathrm{H}), 1.69(\mathrm{~s}, 3 \mathrm{H}), 1.16(\mathrm{~s}, 3 \mathrm{H}) ;{ }^{13} \mathrm{C} \mathrm{NMR}\left(100 \mathrm{MHz}, \mathrm{CDCl}_{3}\right) \delta$ 203.19 (e), 143.76 (e), 142.04 (e), 140.77 (e), 140.24 (e), 134.55 (e), 132.94 (e), 129.75 (o), 128.74 (o), 127.91 (o), 127.69 (o), 115.04 (o), 61.56 (e), 49.86 (e), 42.44 (e), 41.27 (e), 30.17 (o), 21.54 (o), 21.34 (o); HRMS (CI, $\mathrm{M}+\mathrm{H}^{+}$) calcd for $\mathrm{C}_{24} \mathrm{H}_{26} \mathrm{O}_{3} \mathrm{~N}_{1} \mathrm{~S}_{1}$ 408.1628, found 408.1632 . 


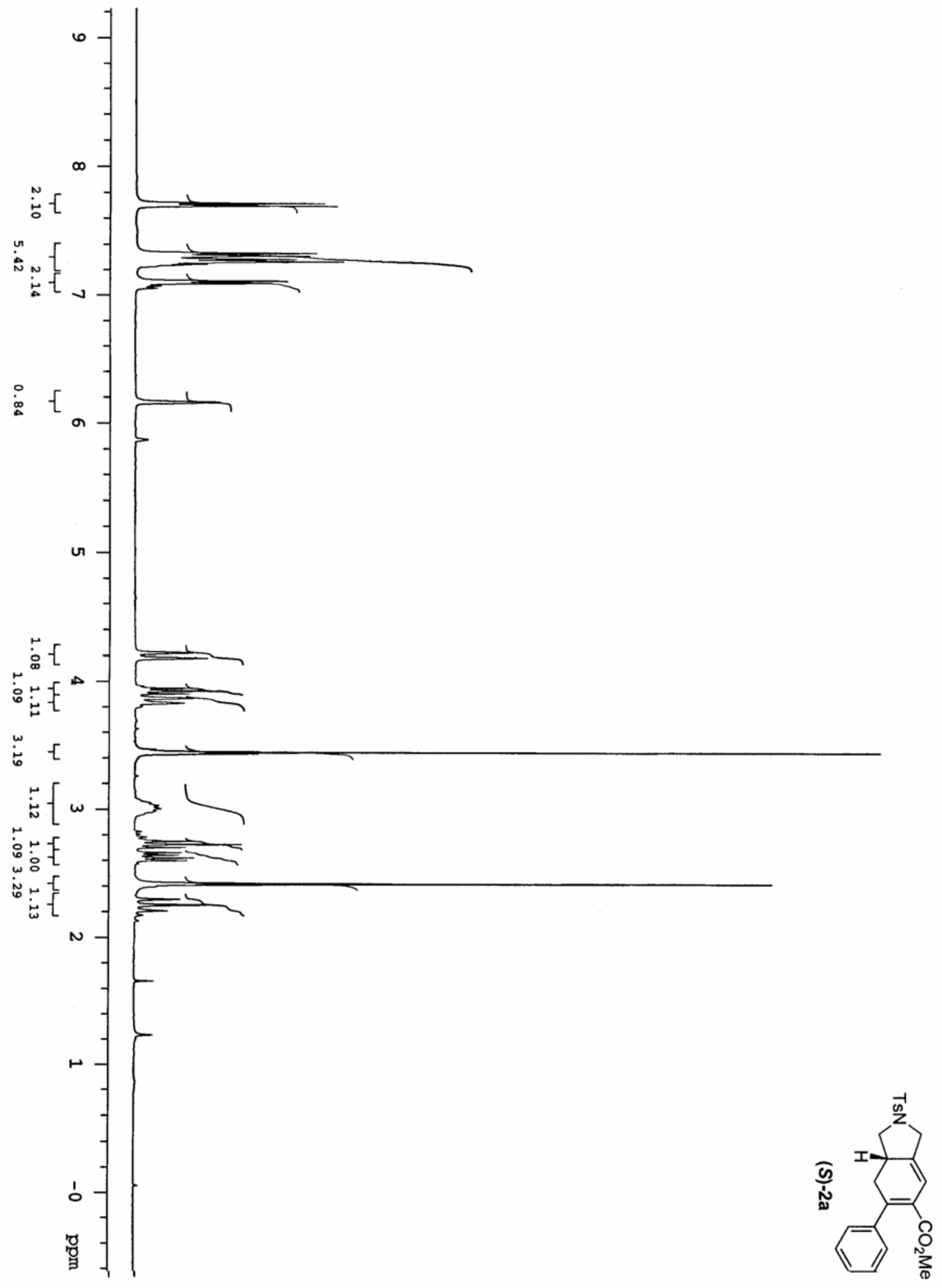




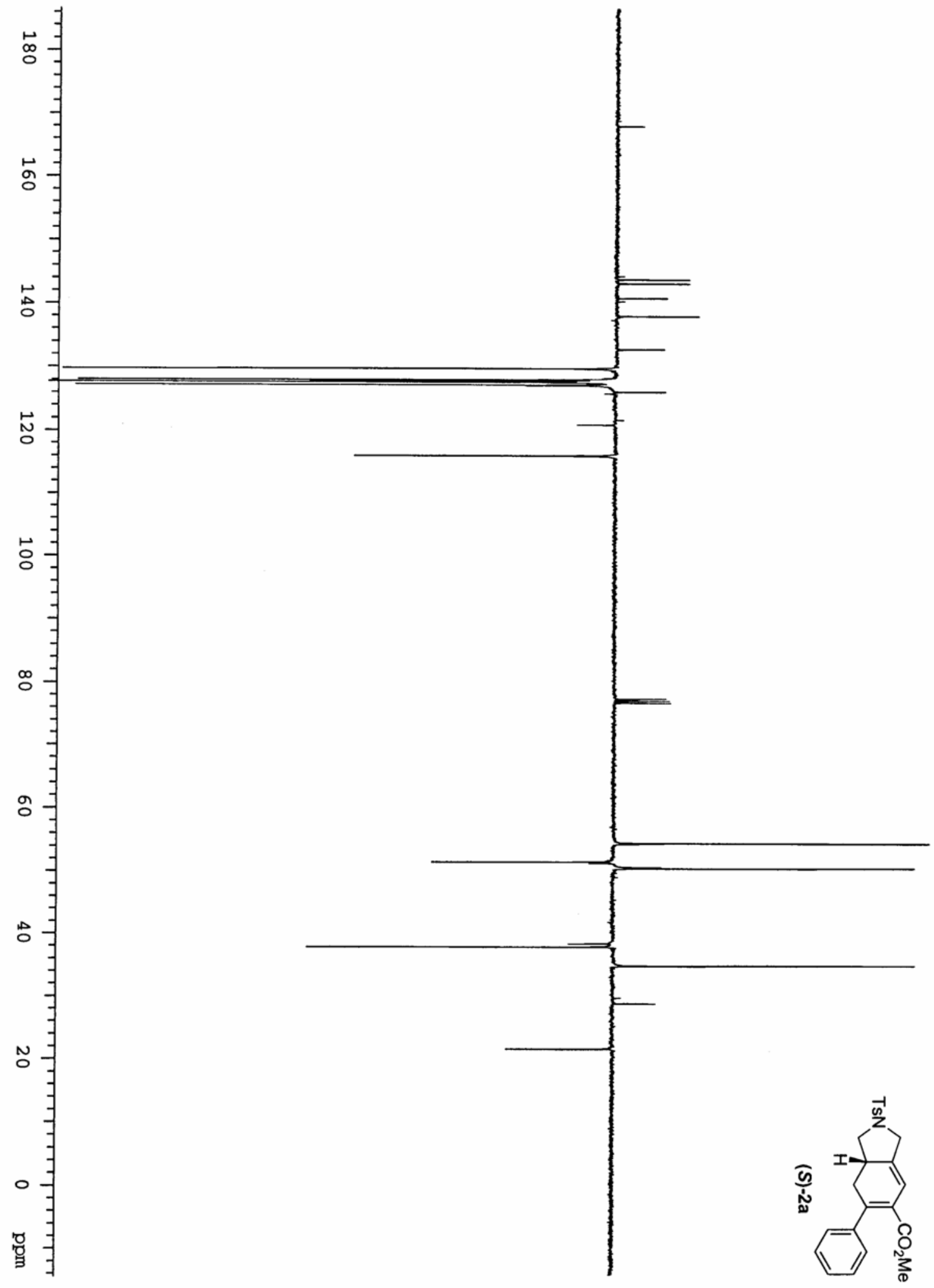




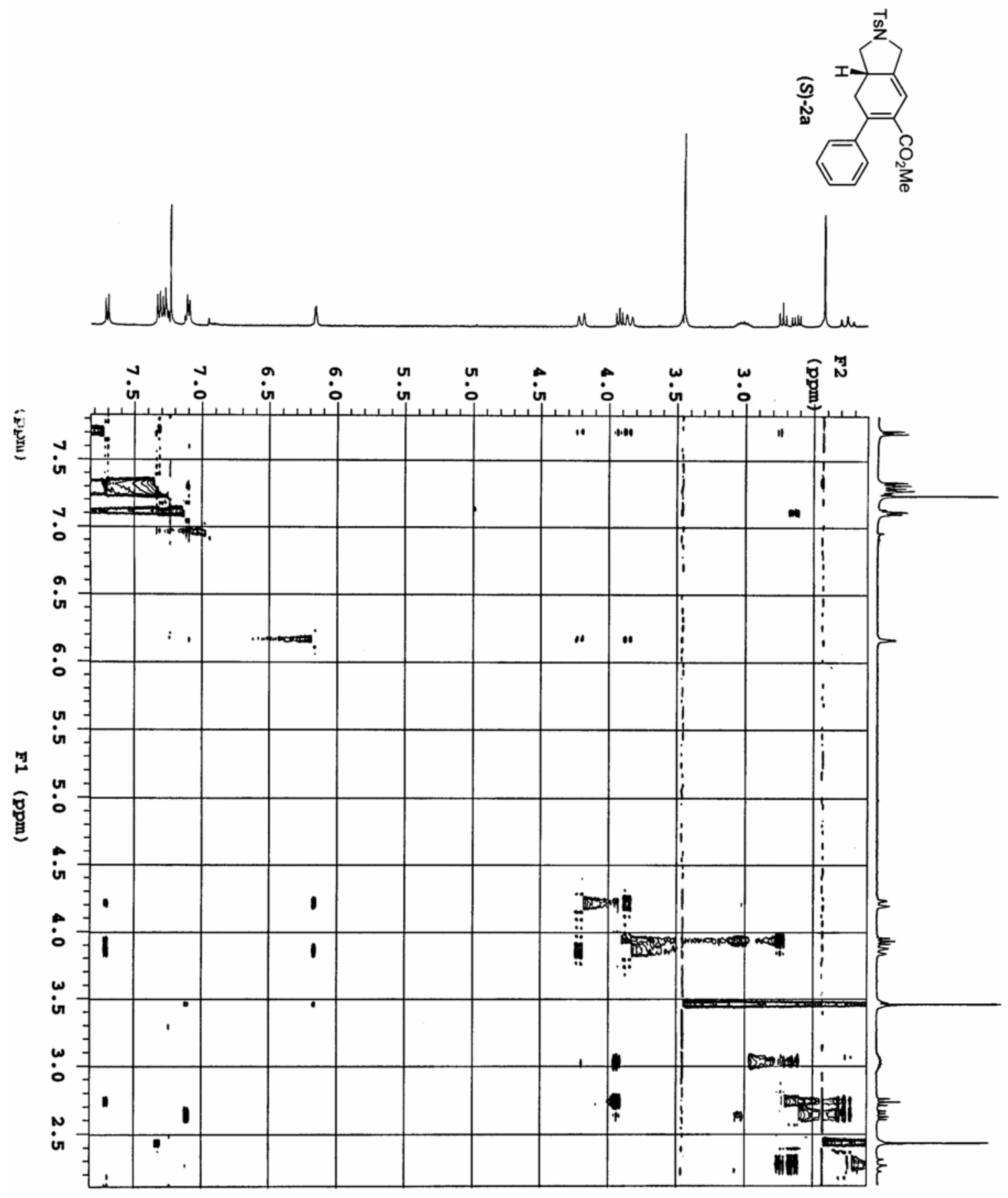




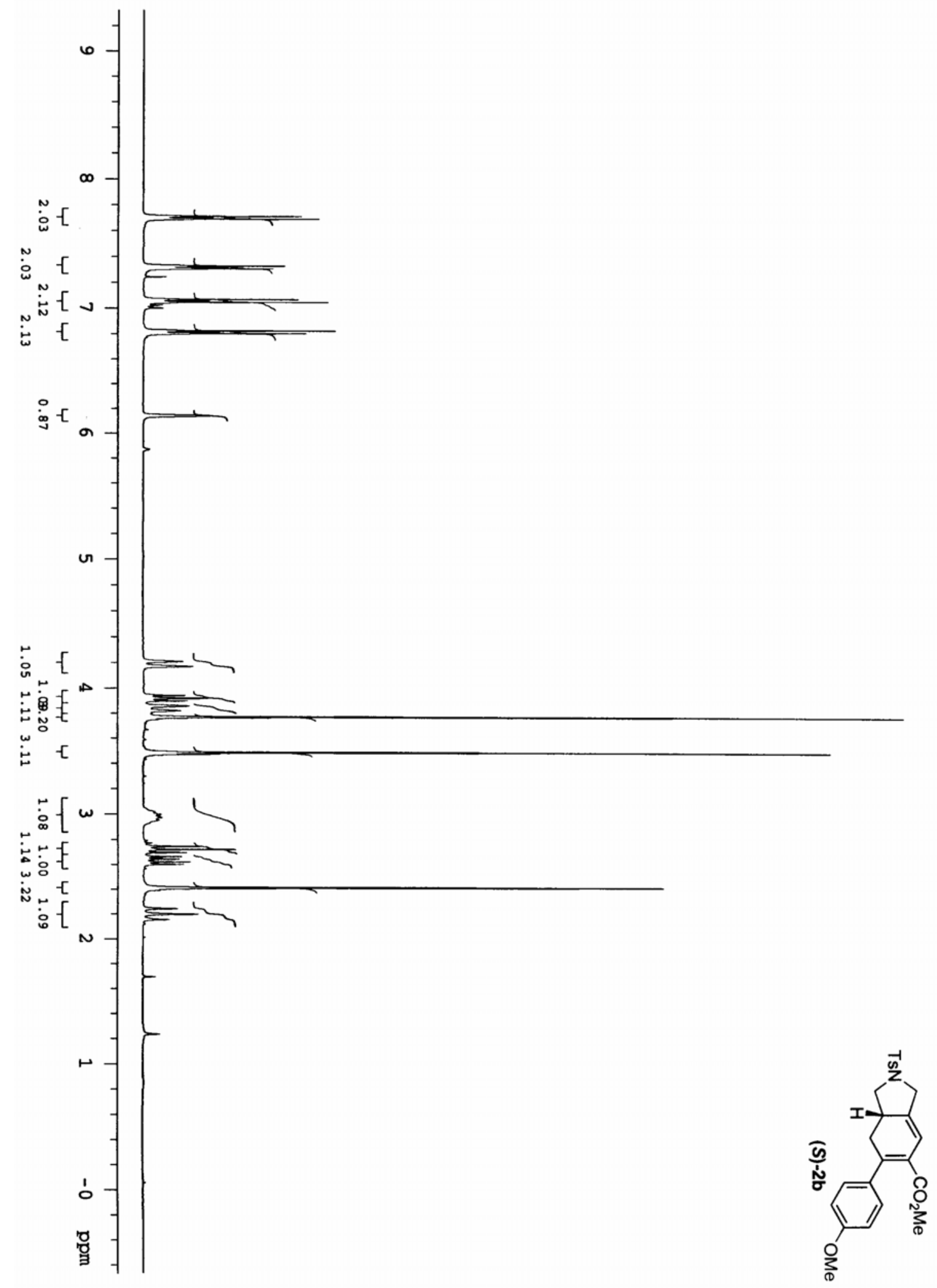




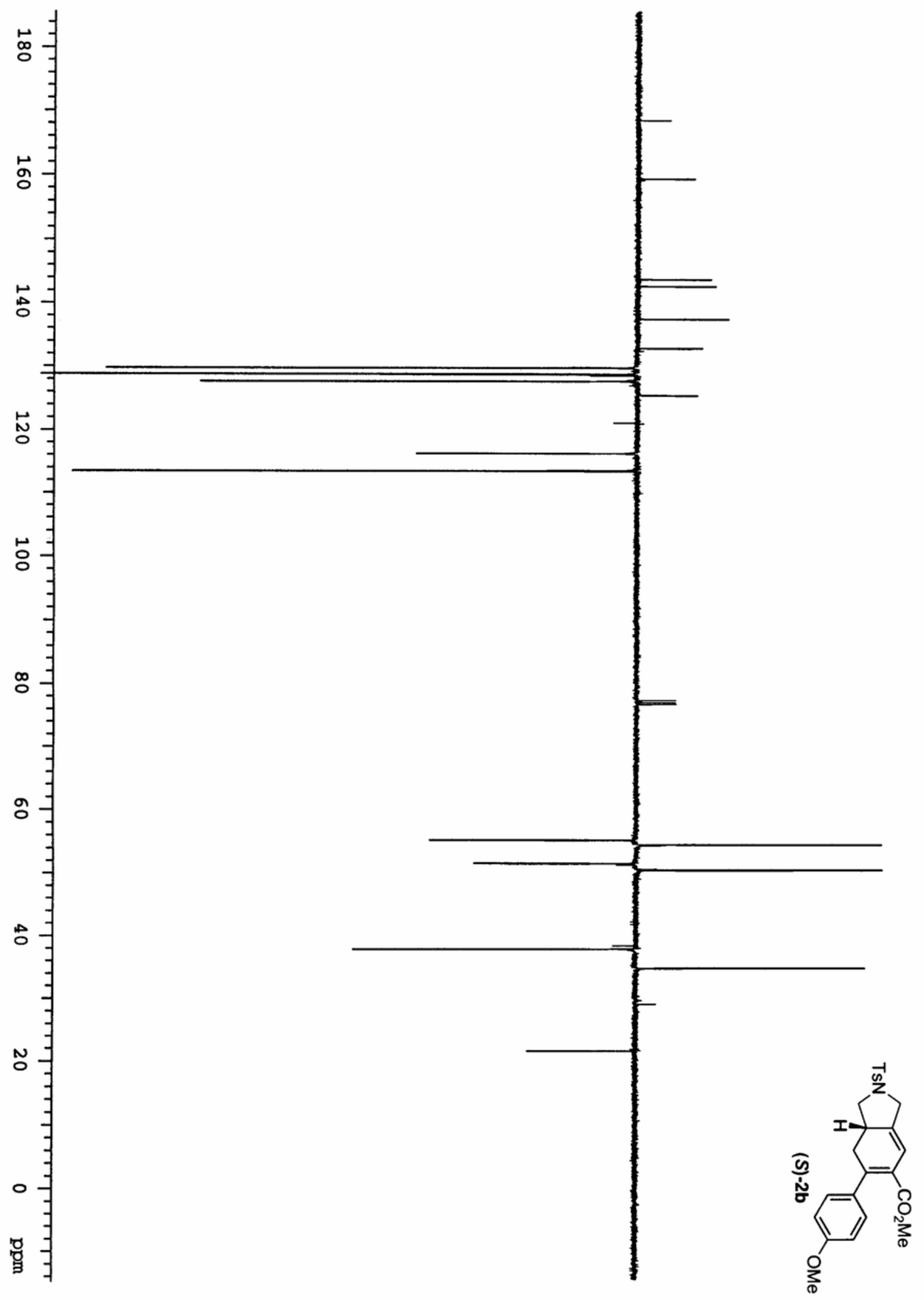



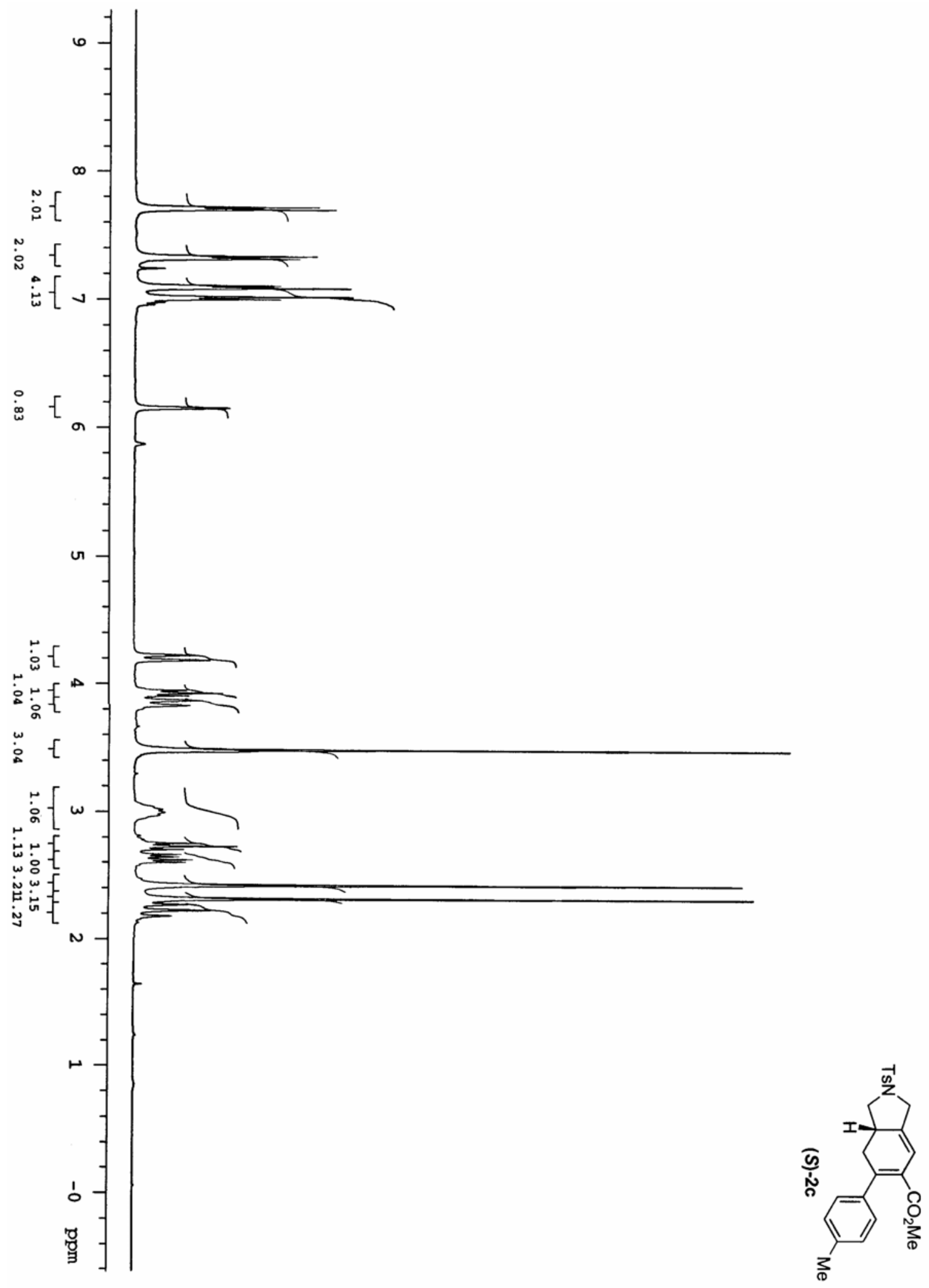


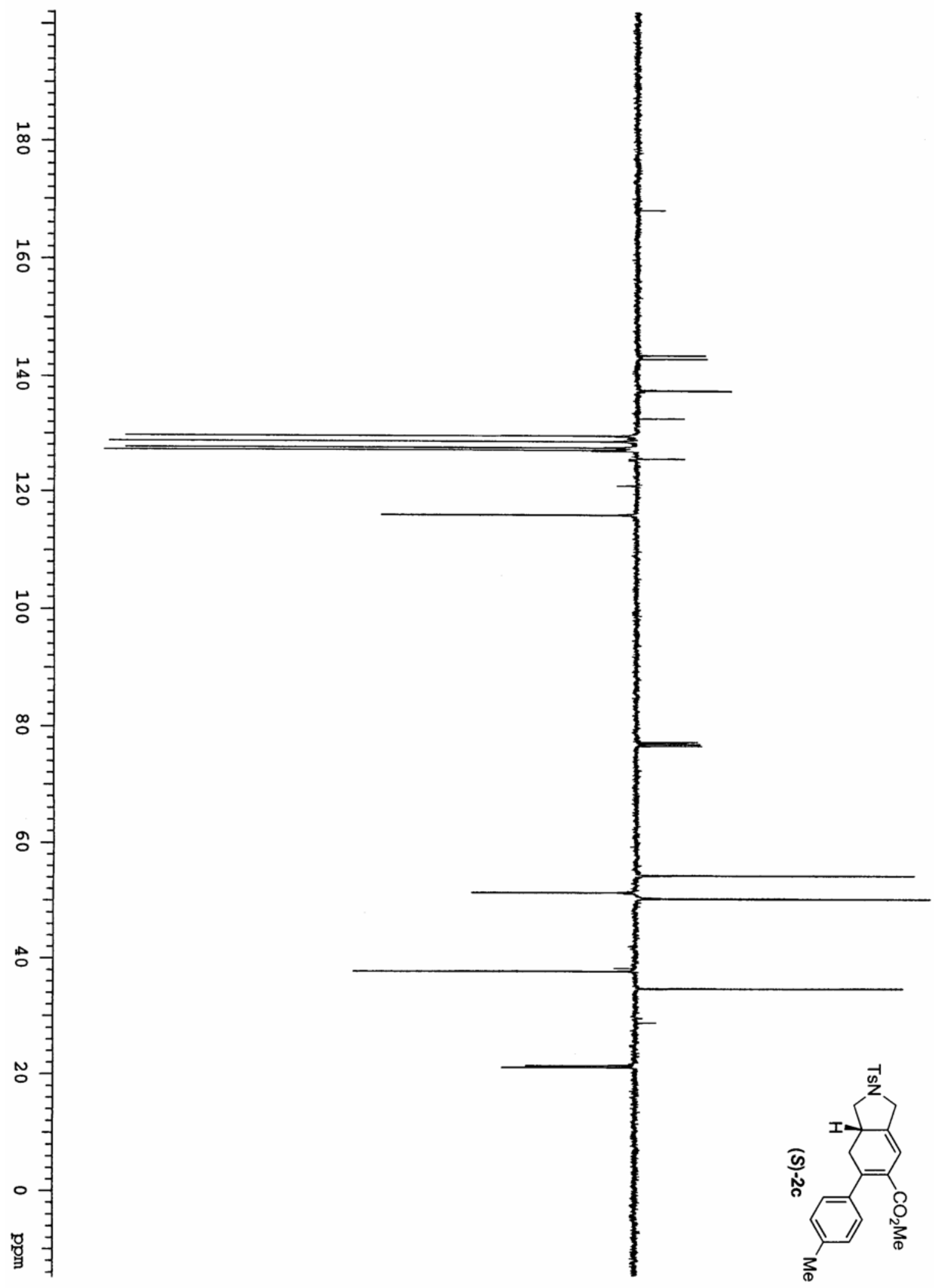




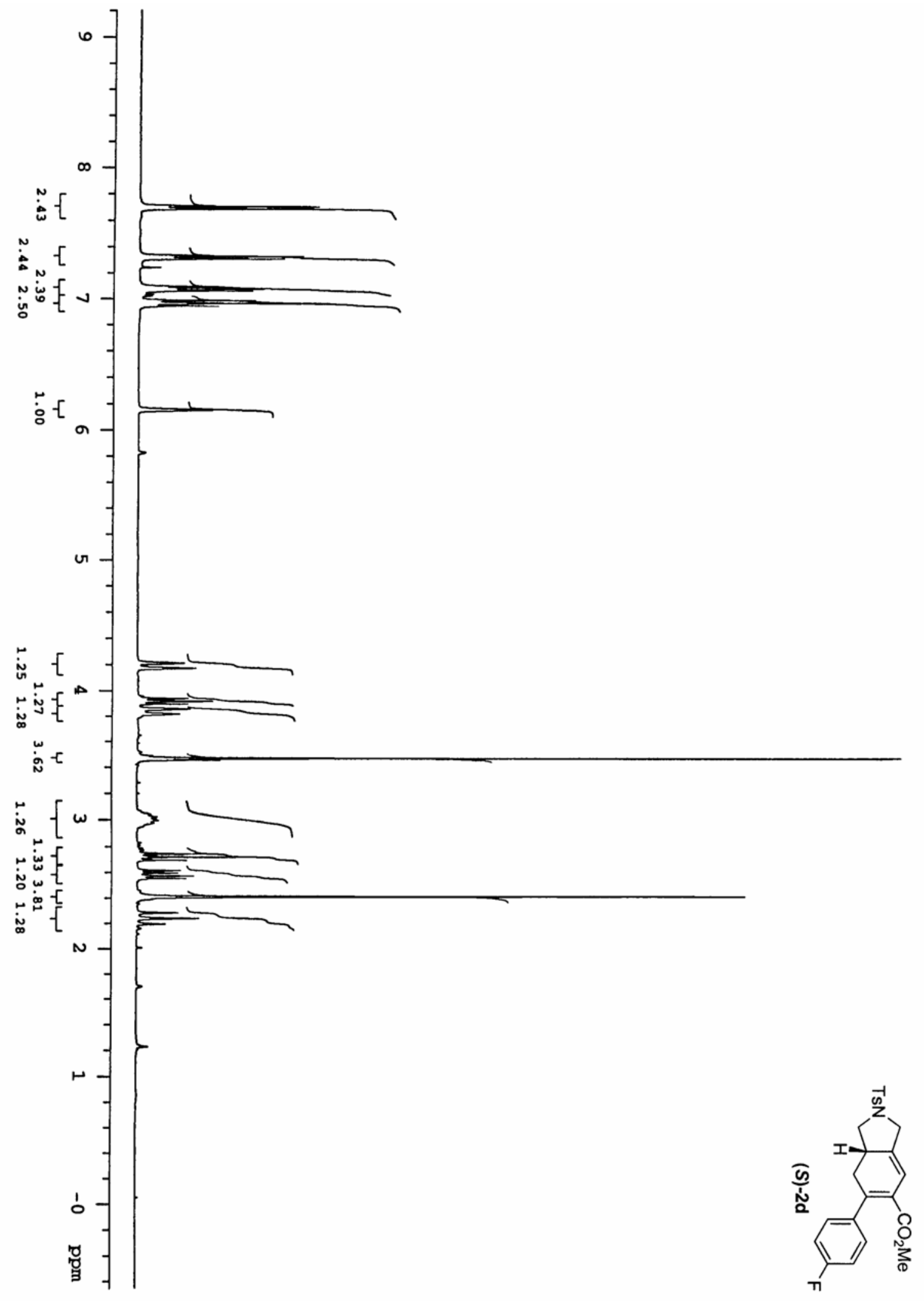




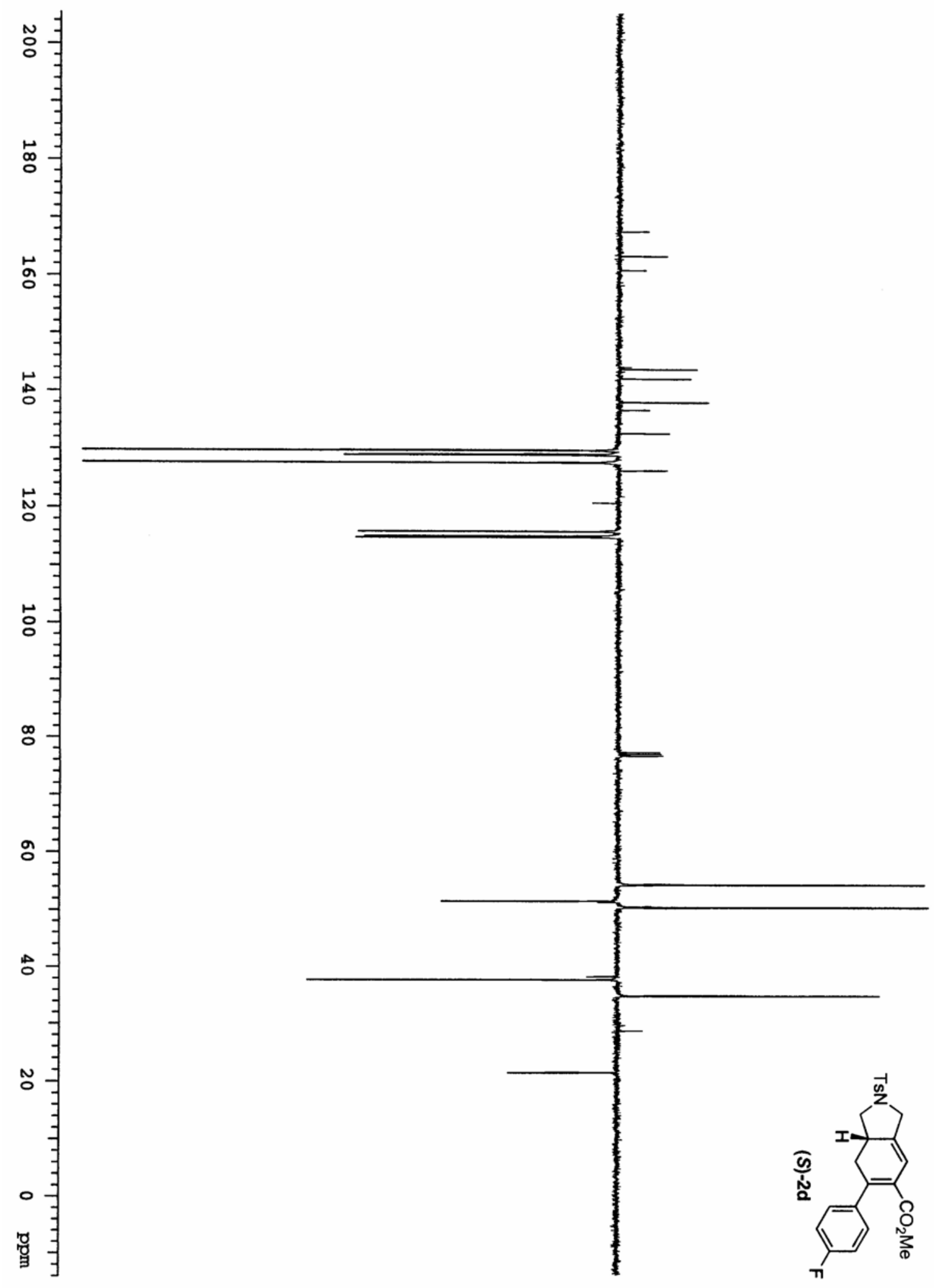




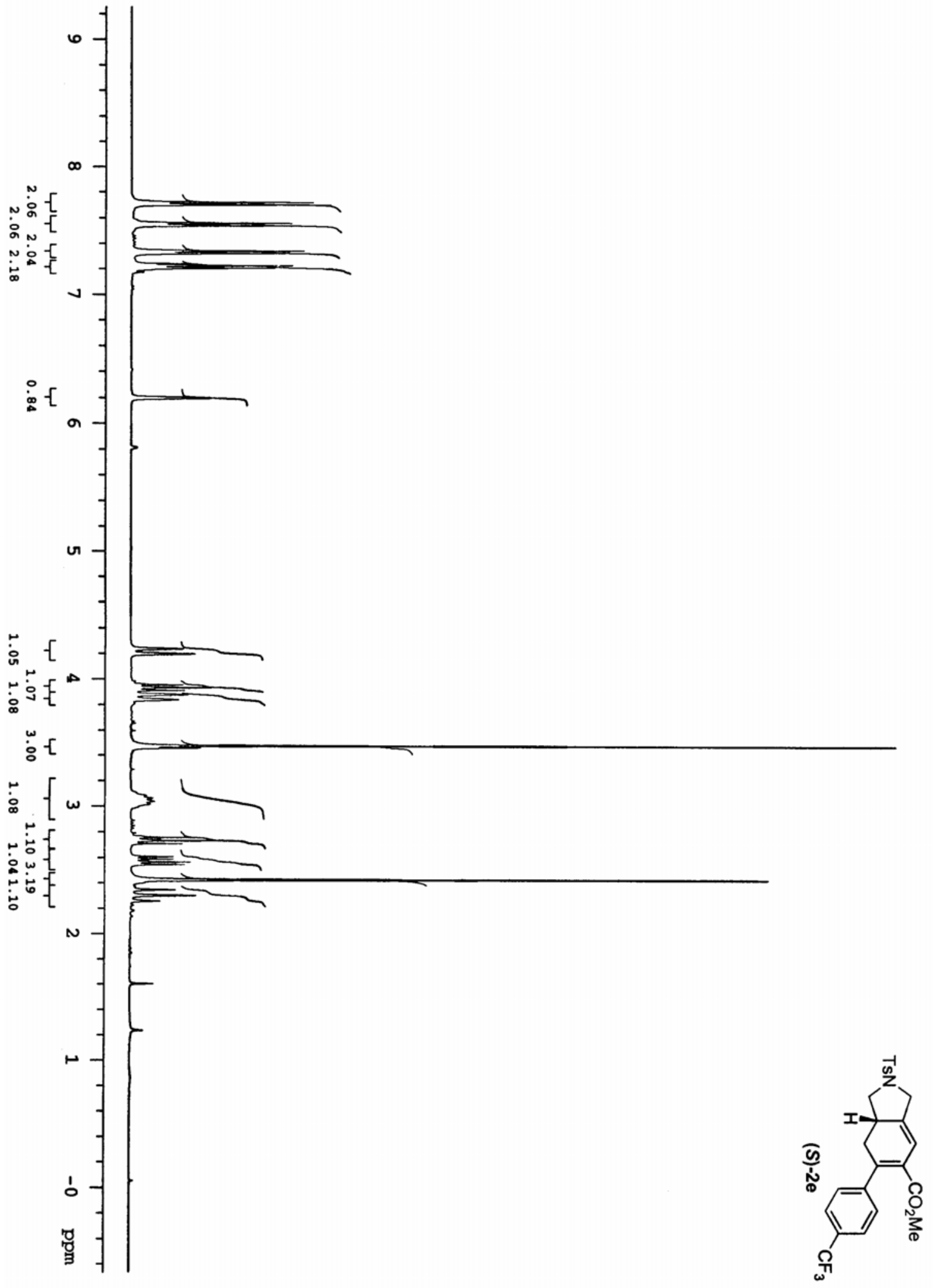




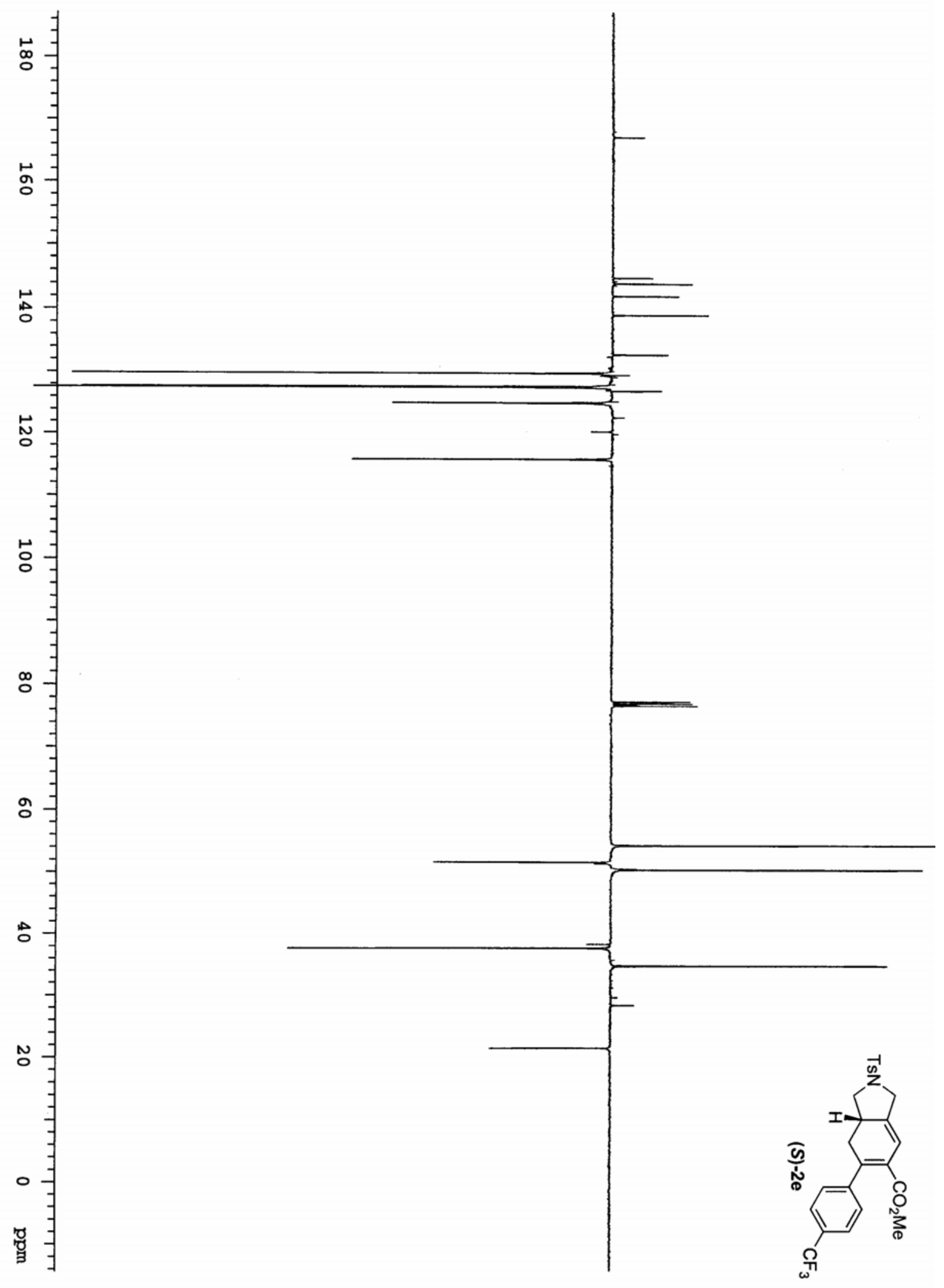




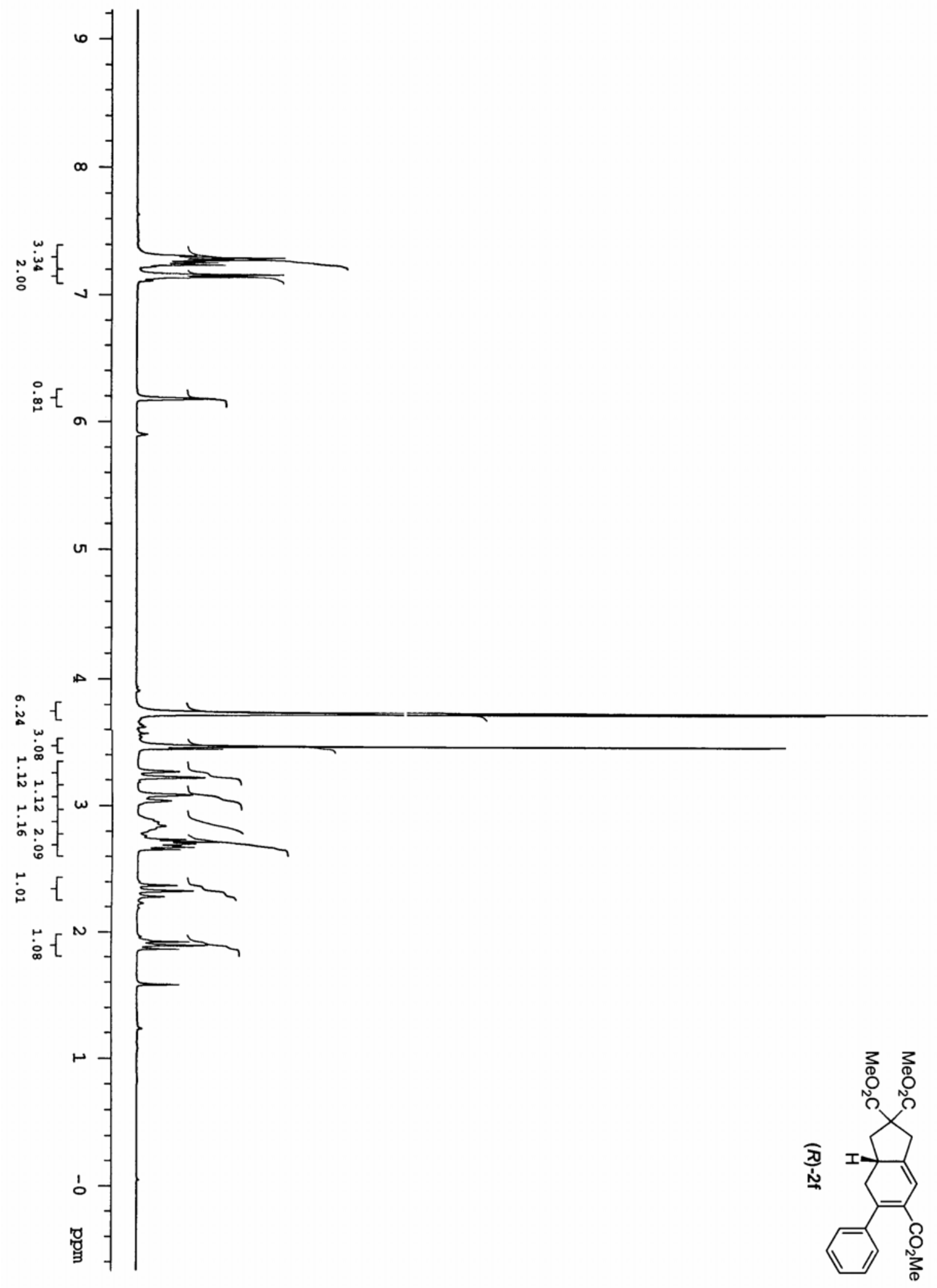




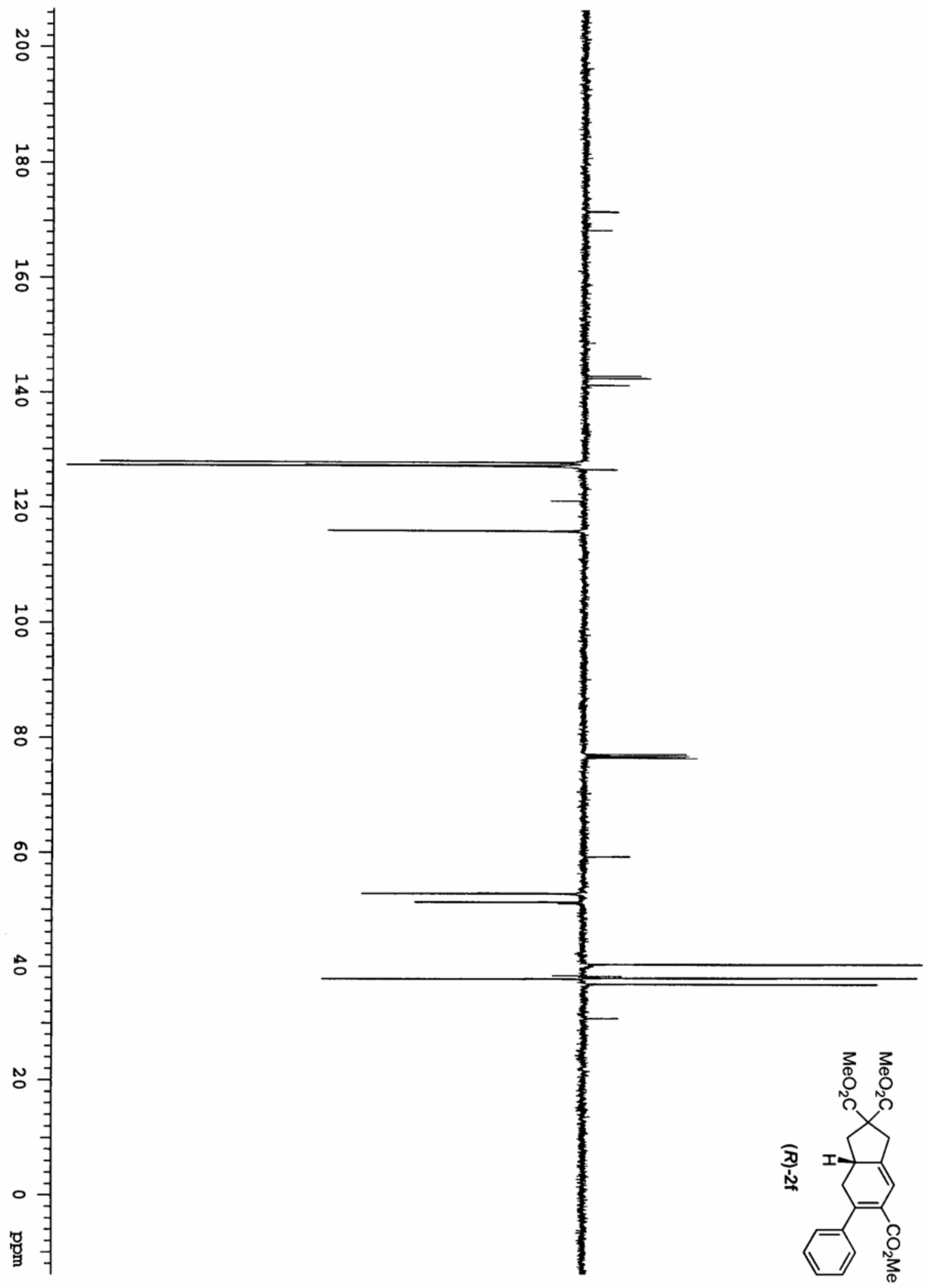




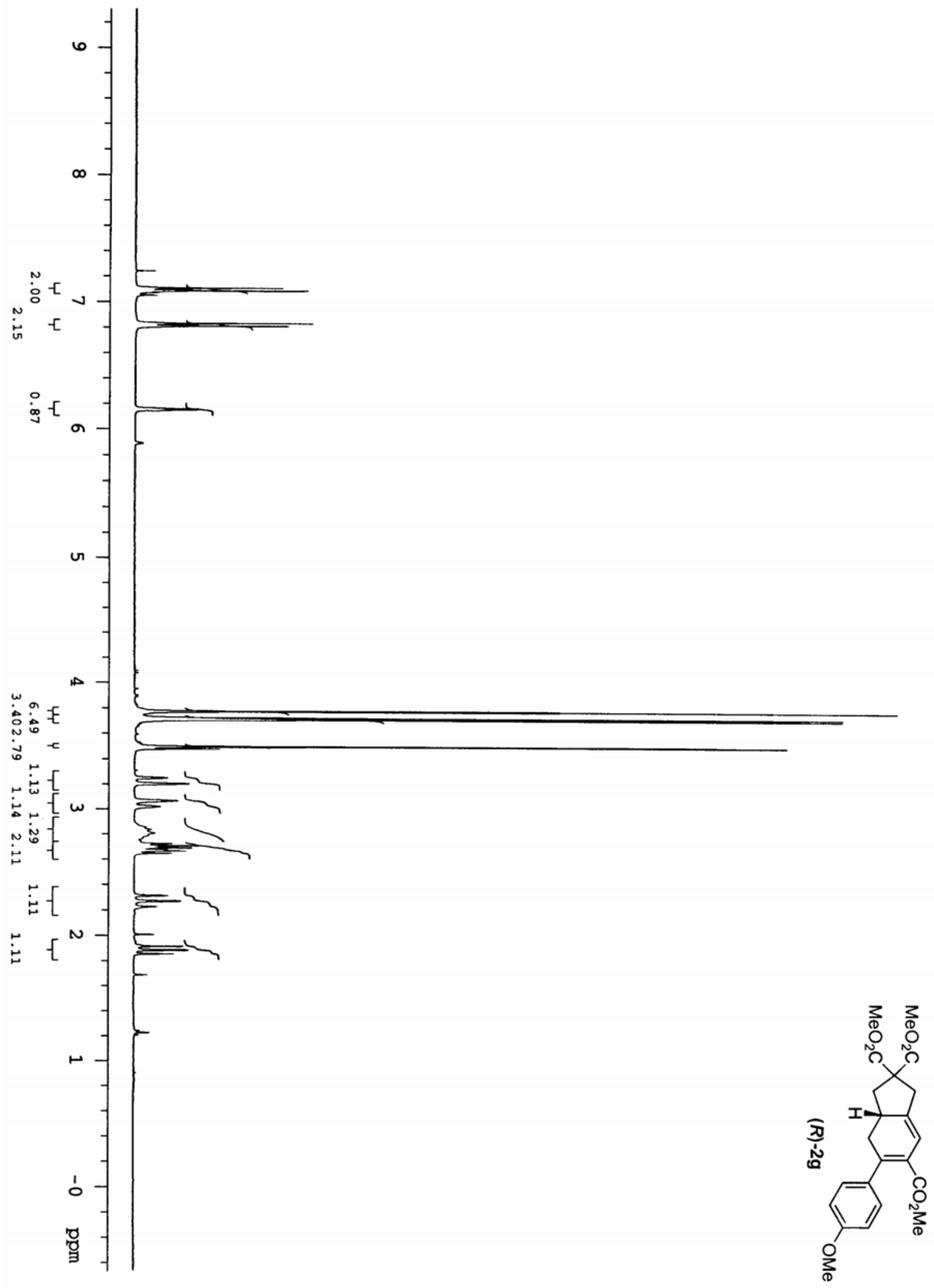




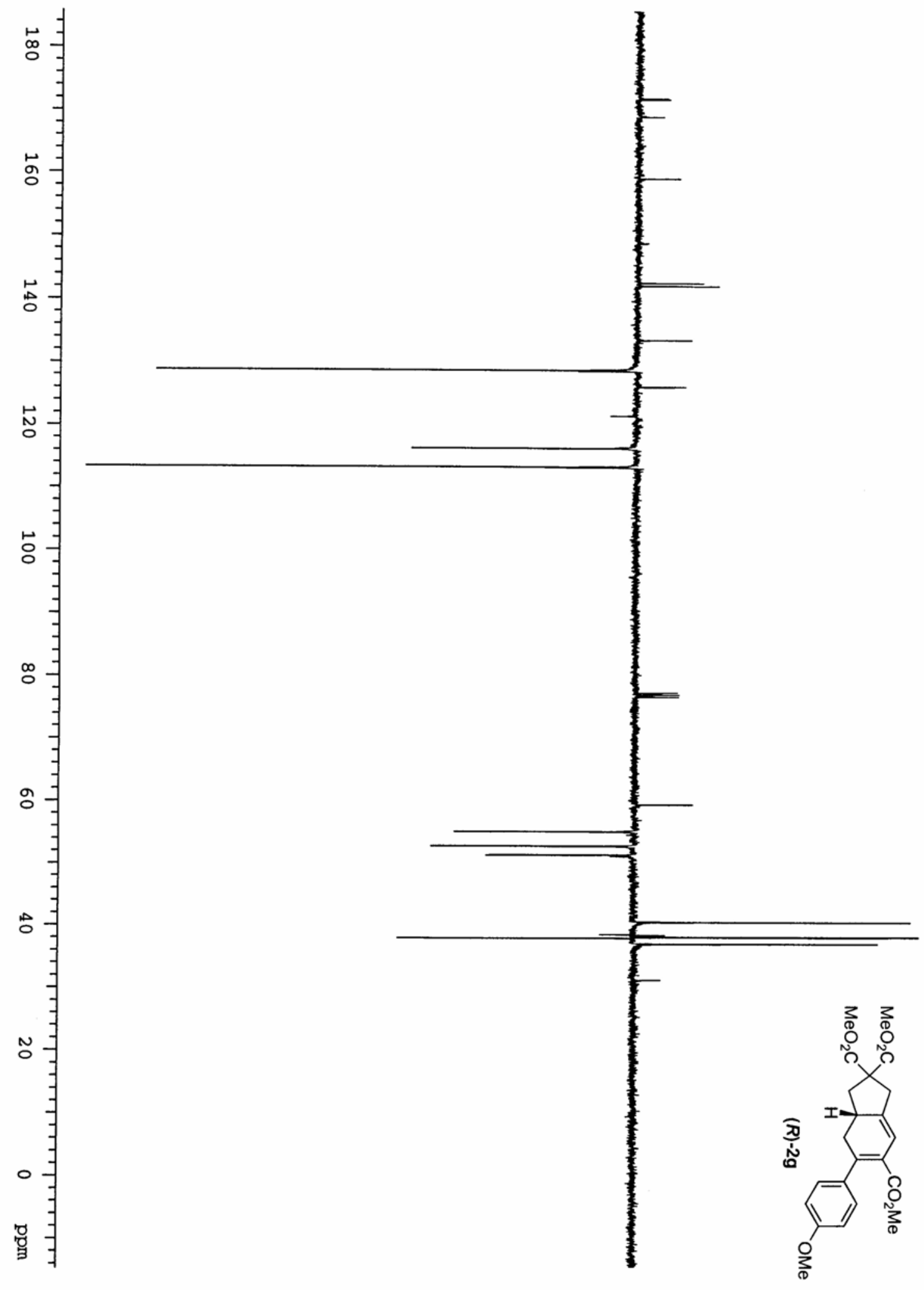




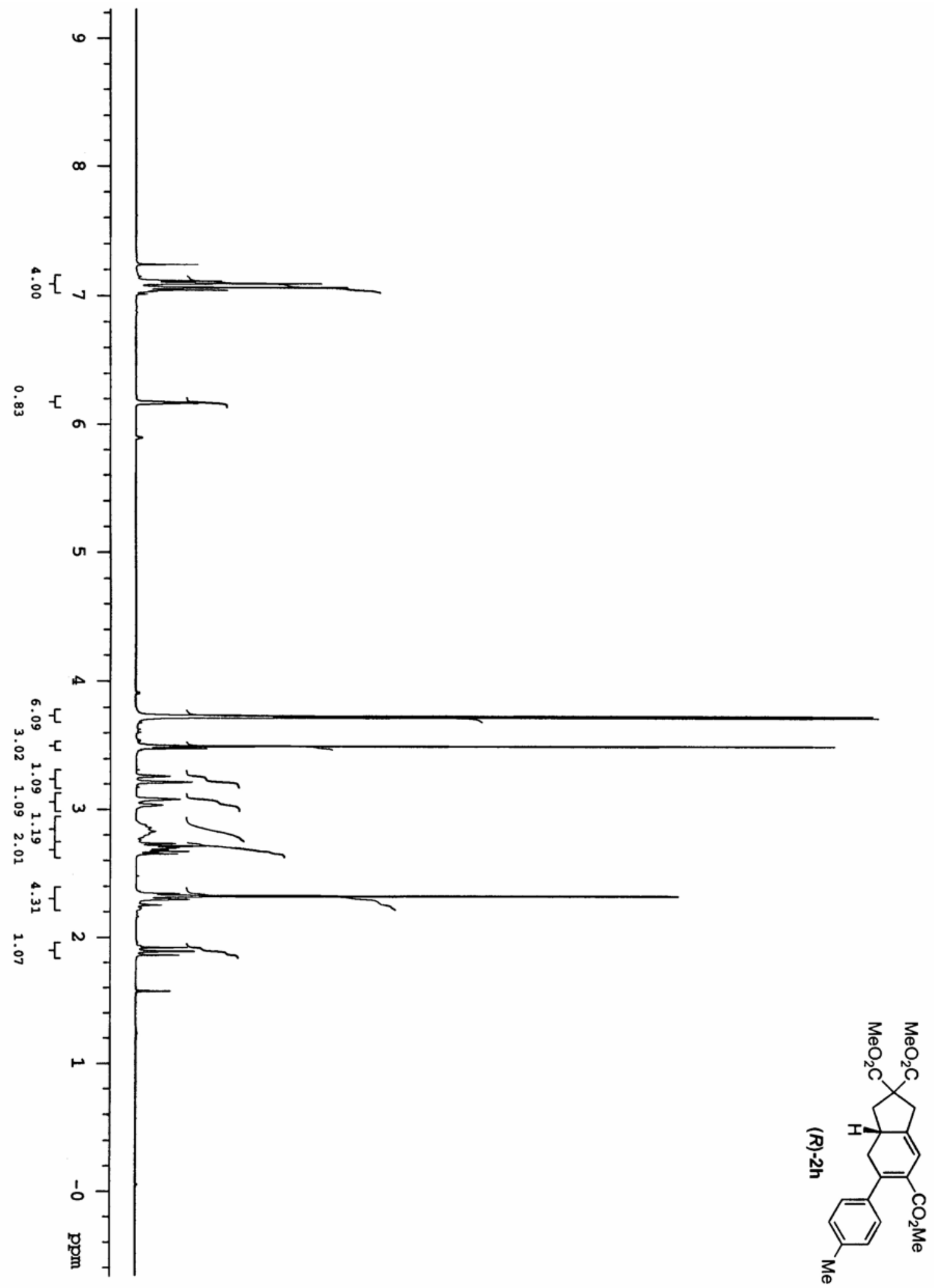




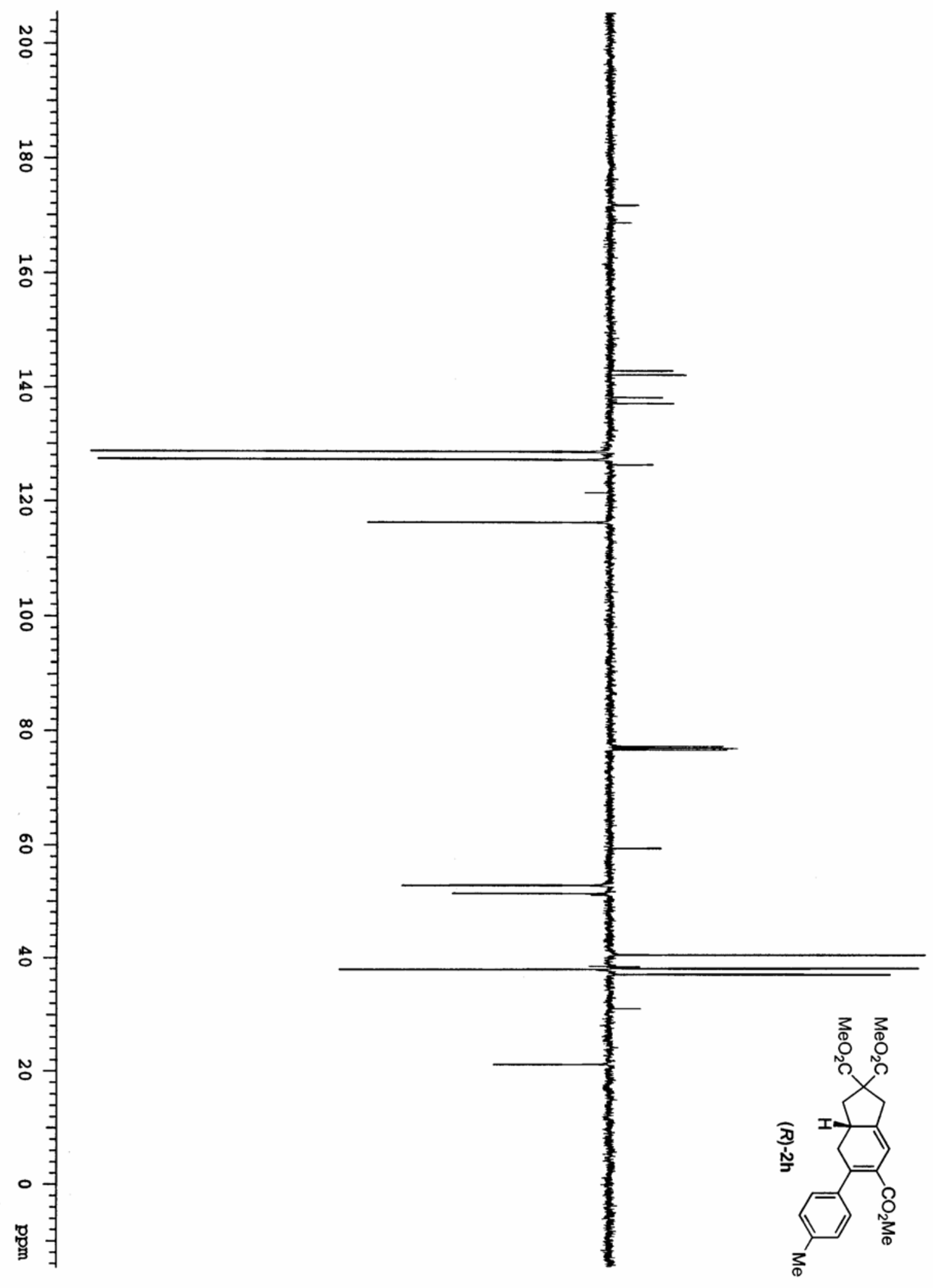




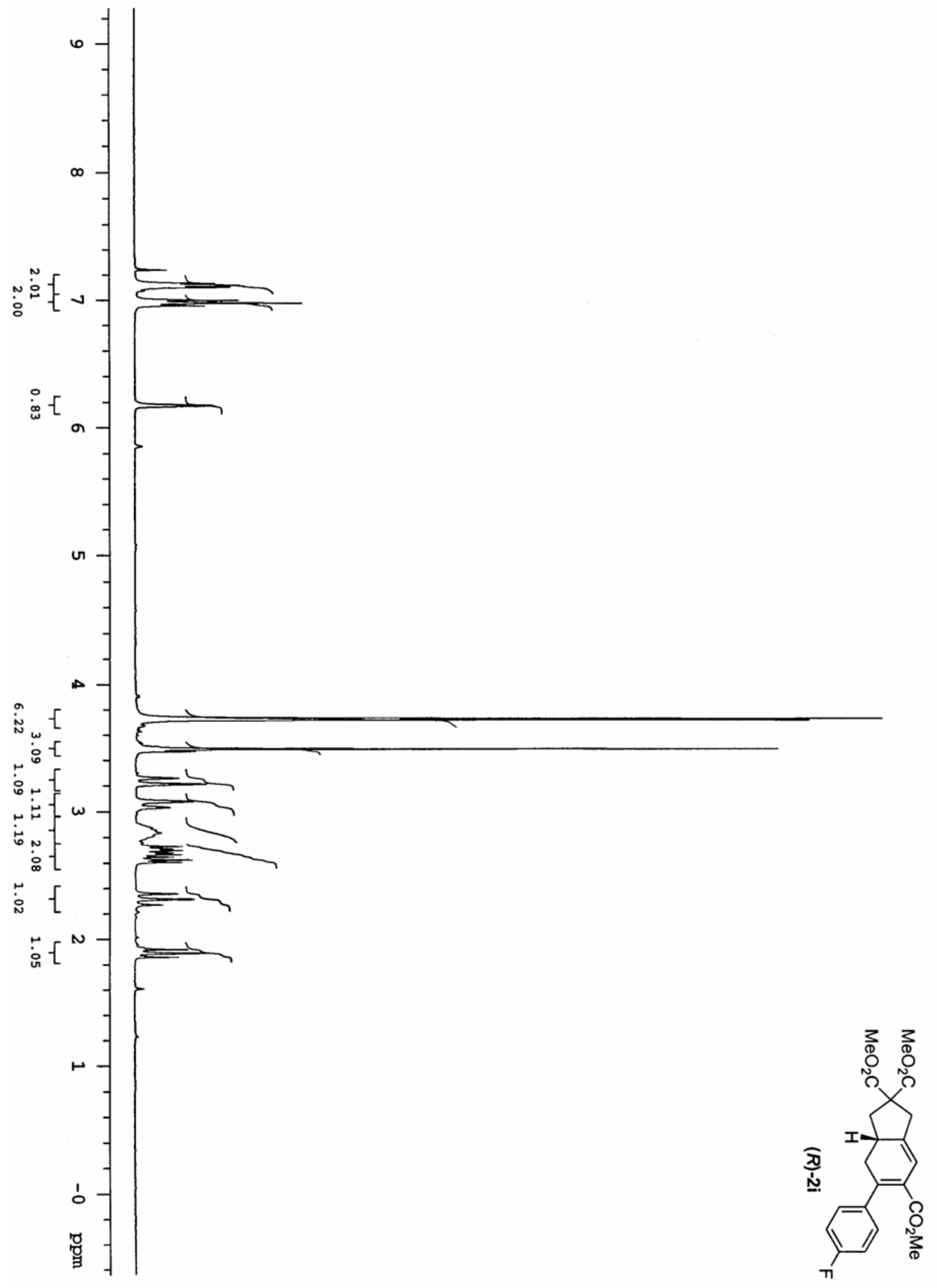




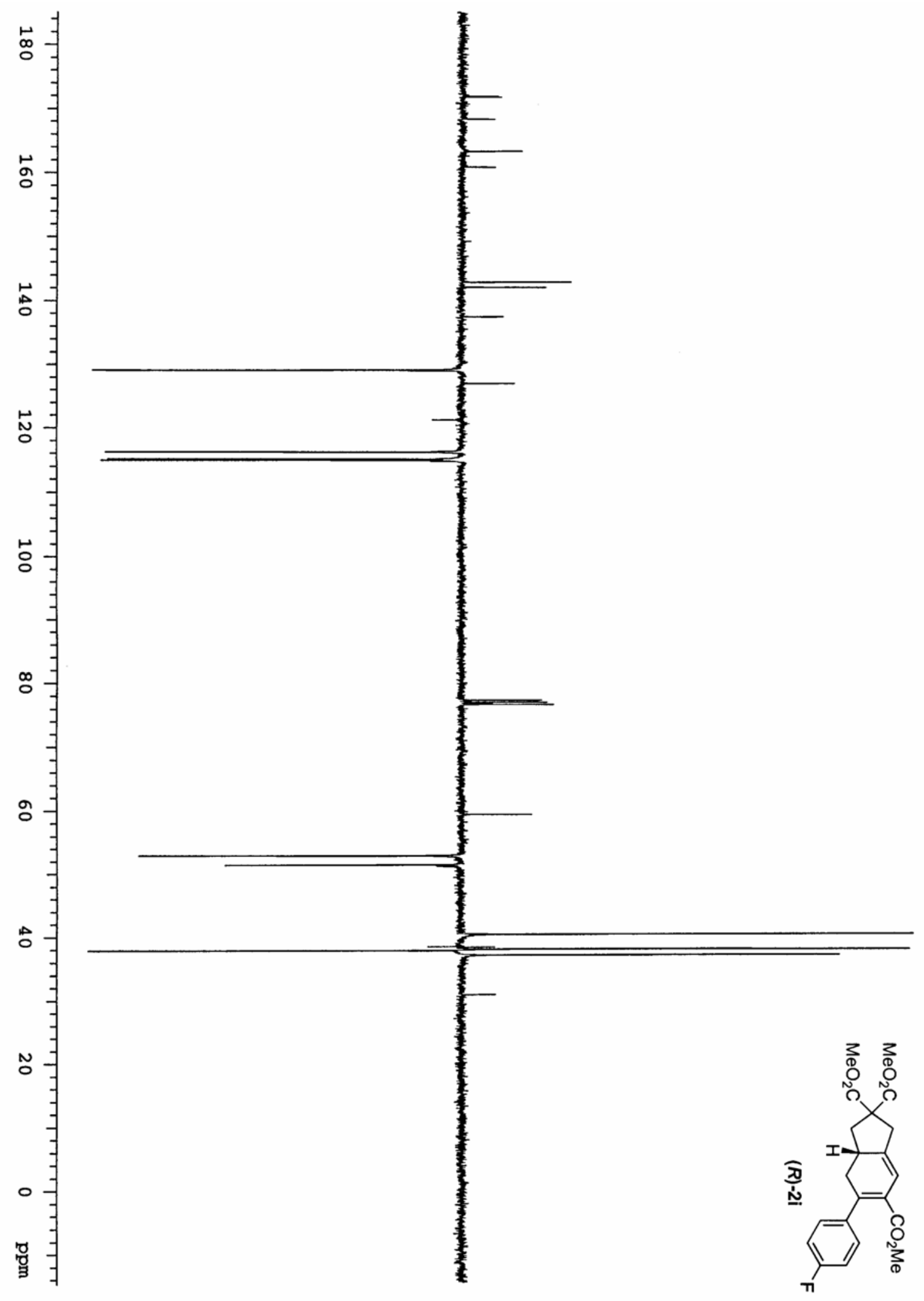




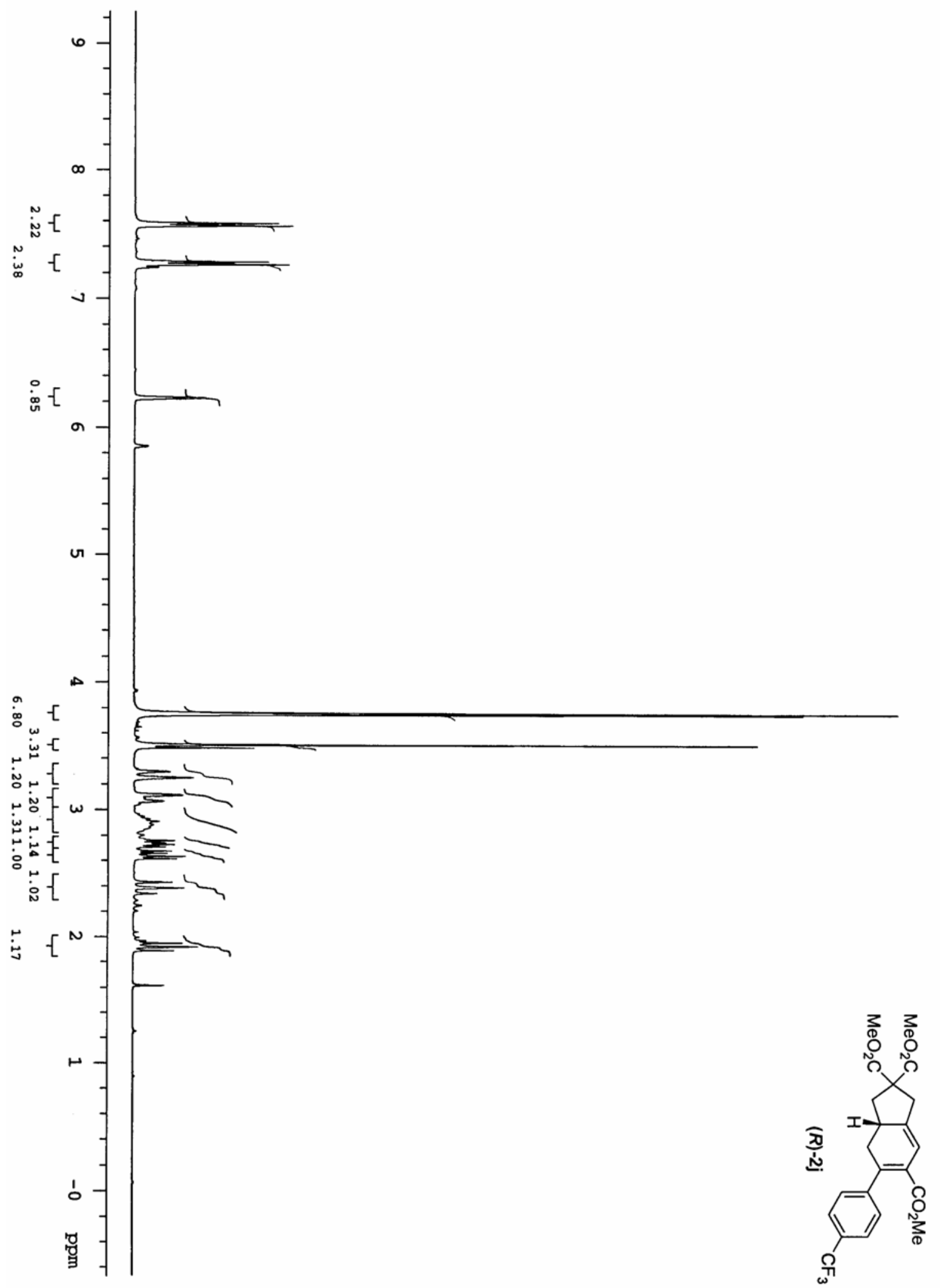




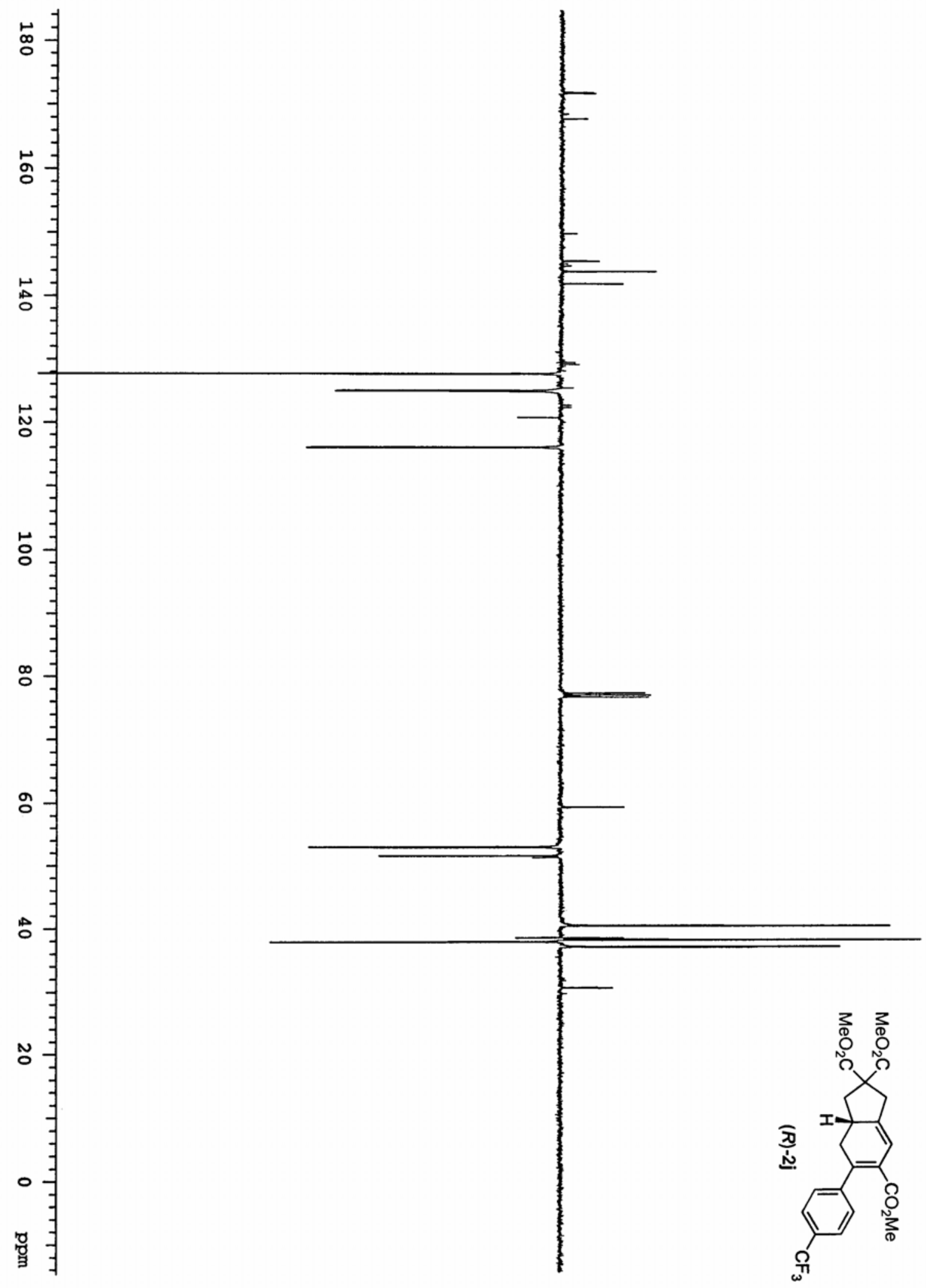




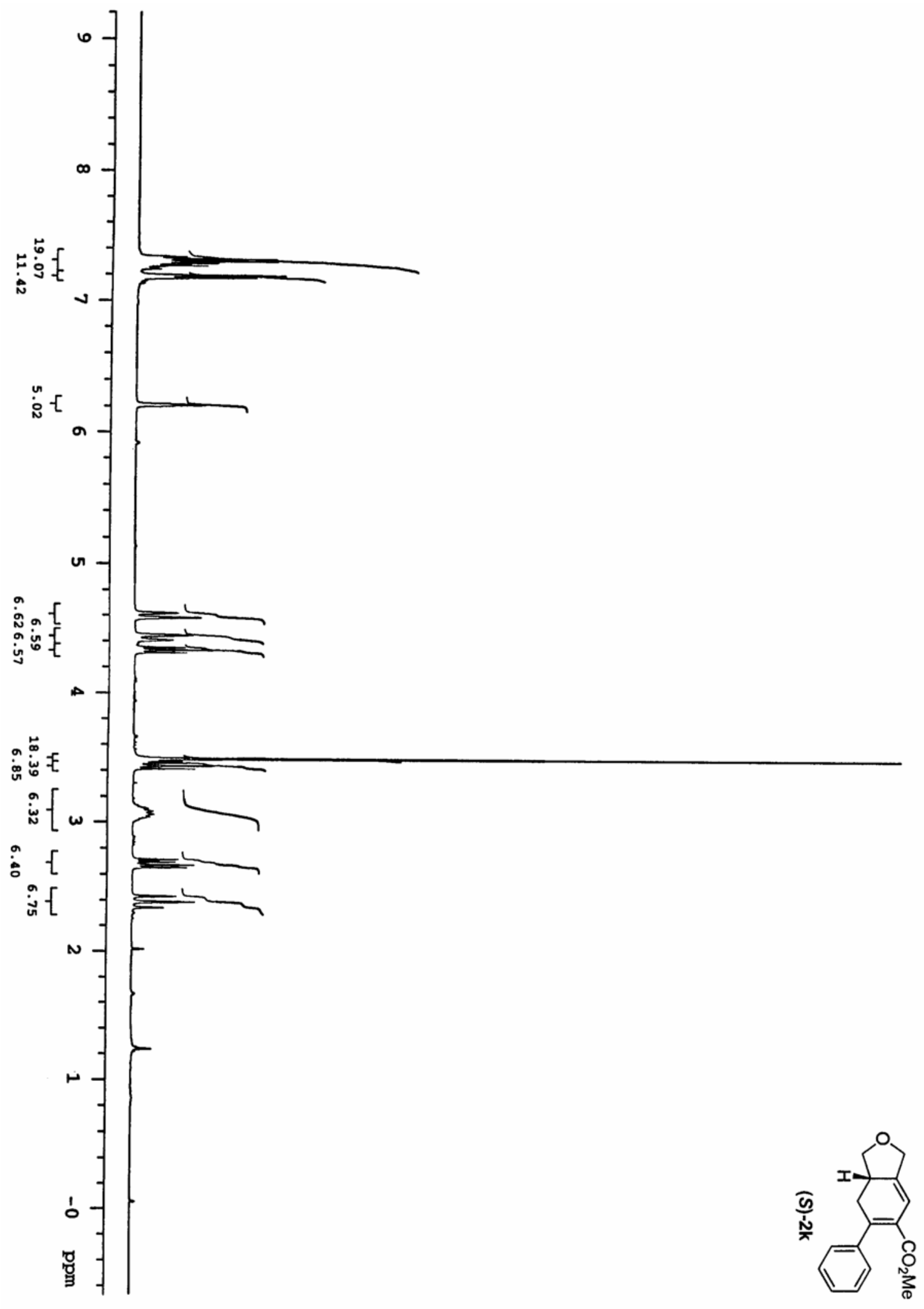



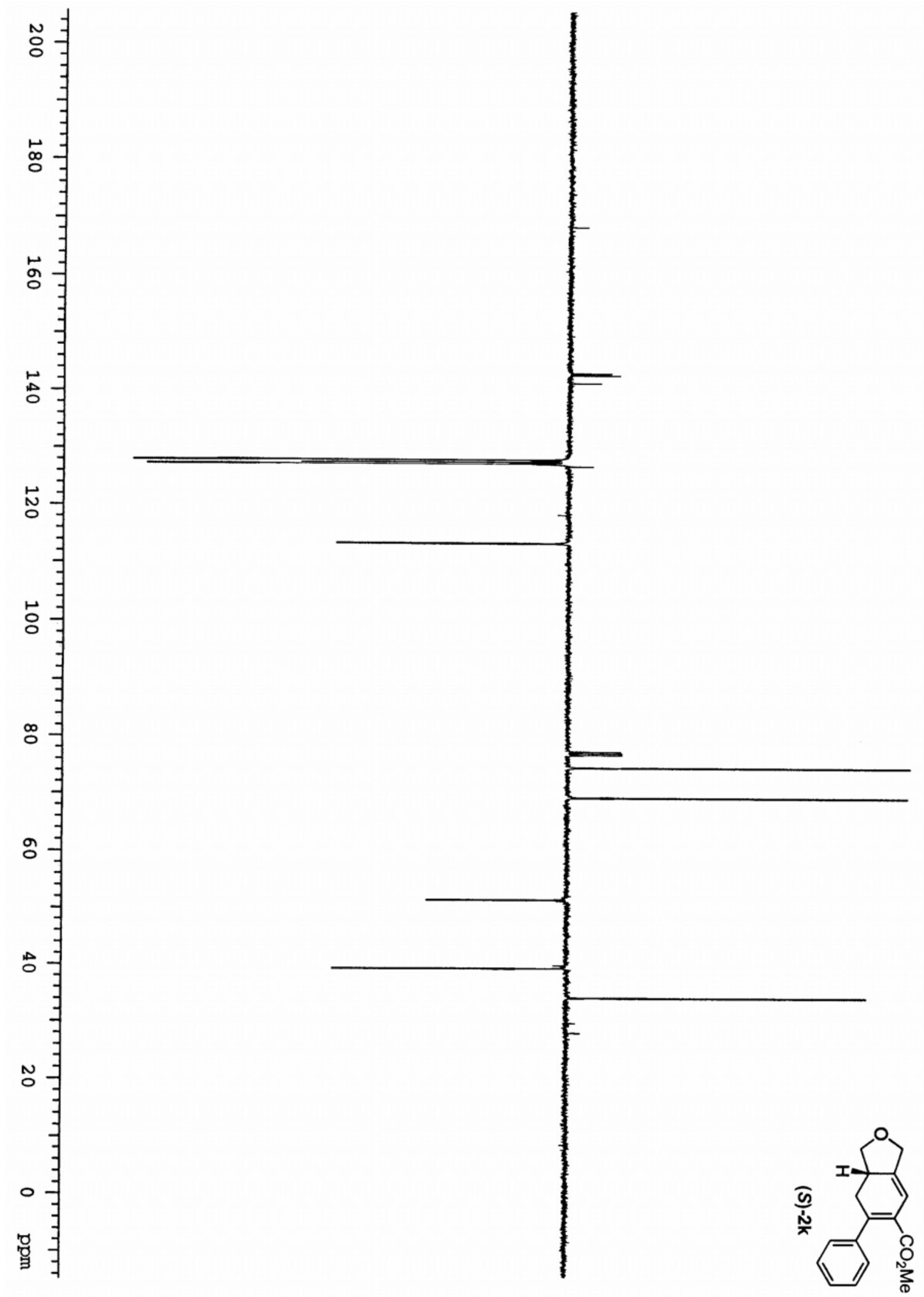


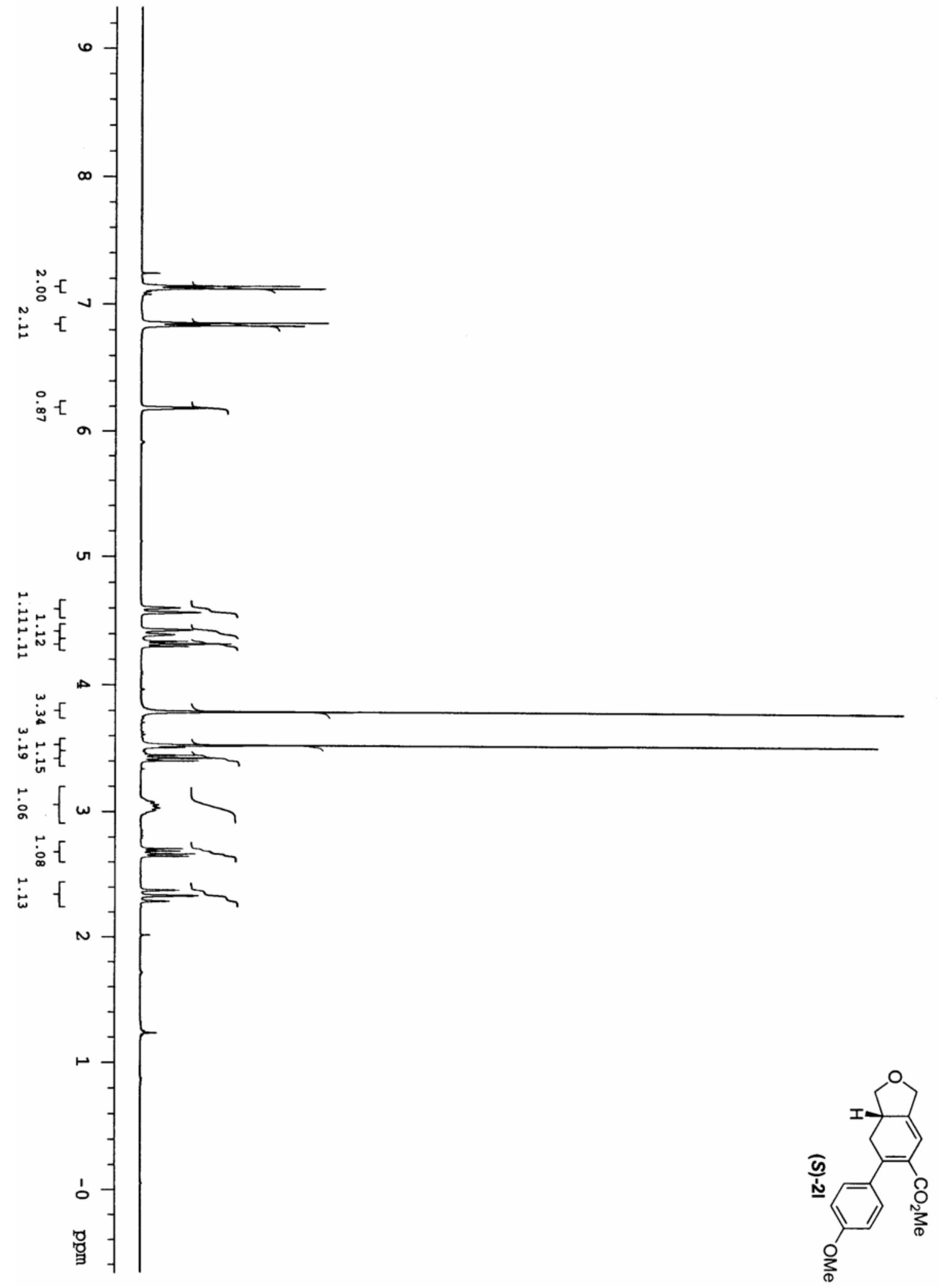




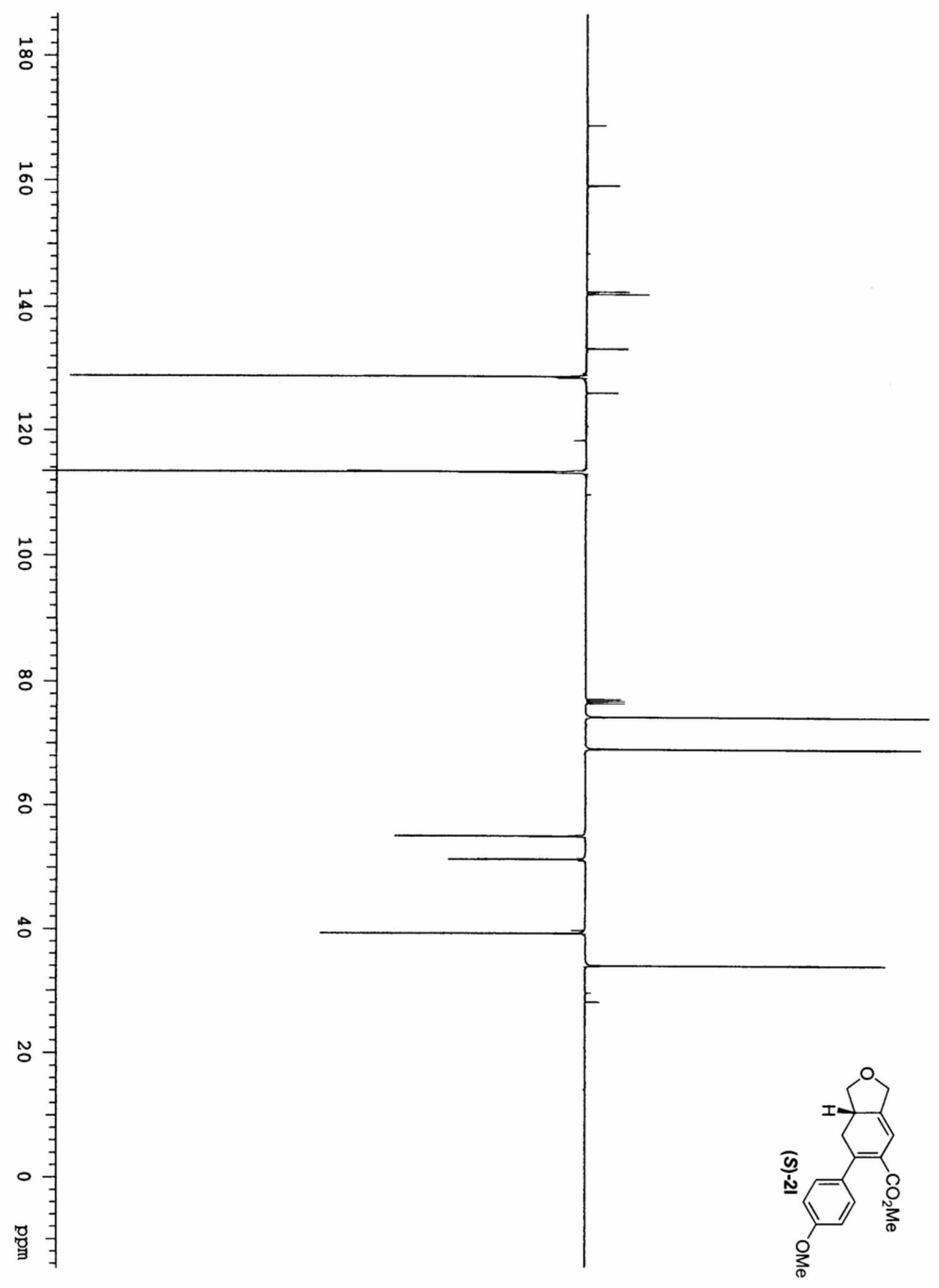




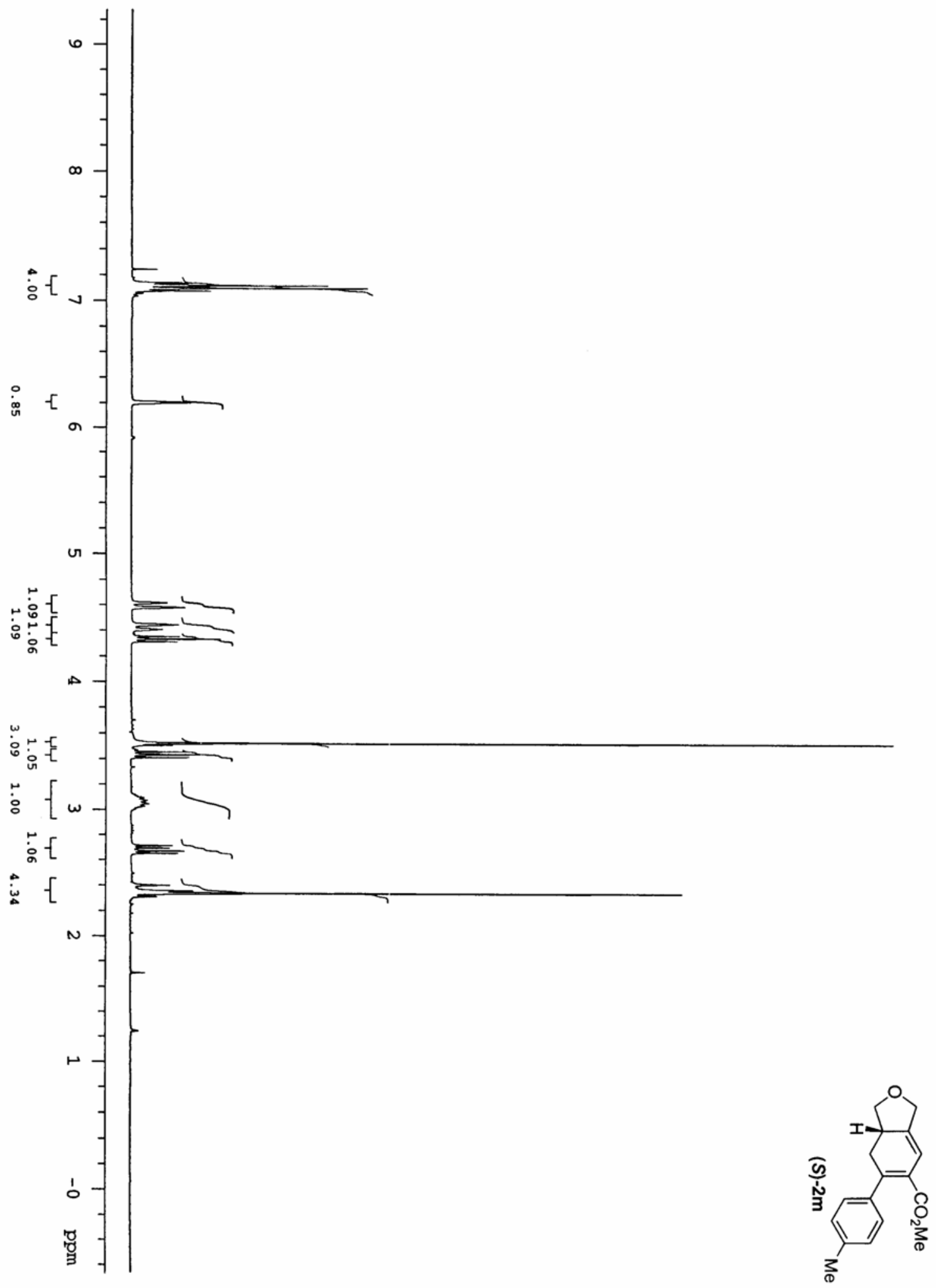




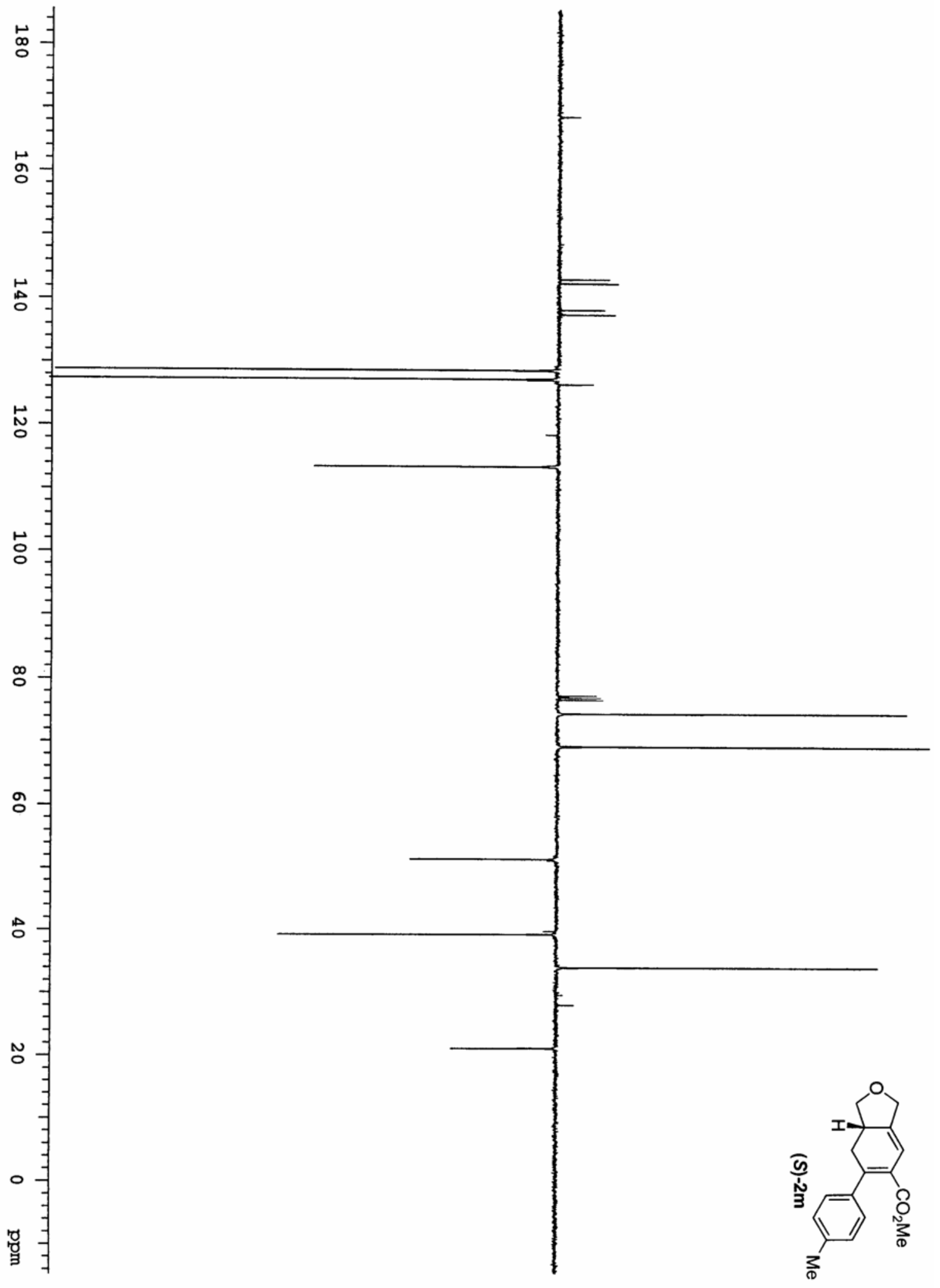




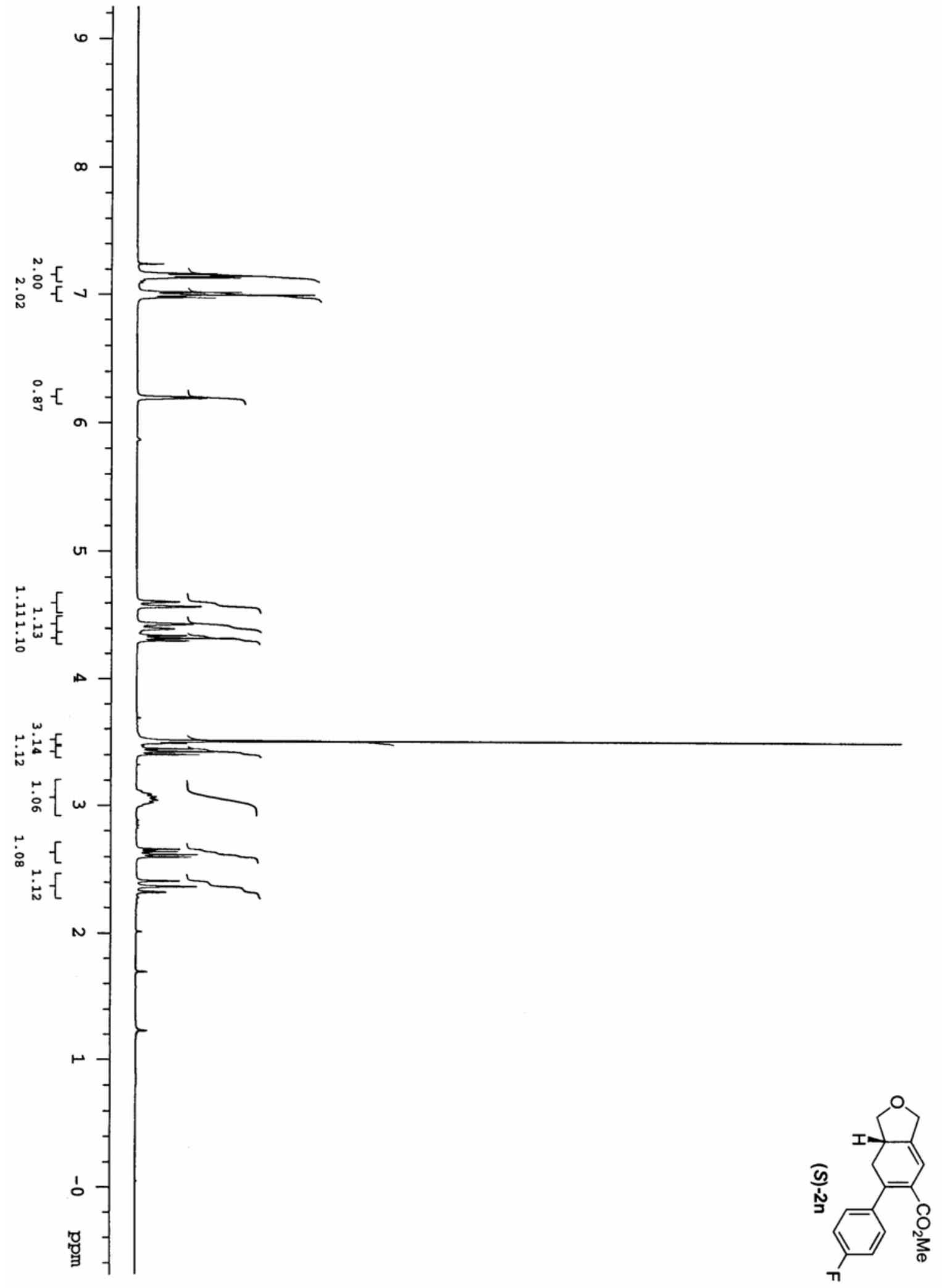




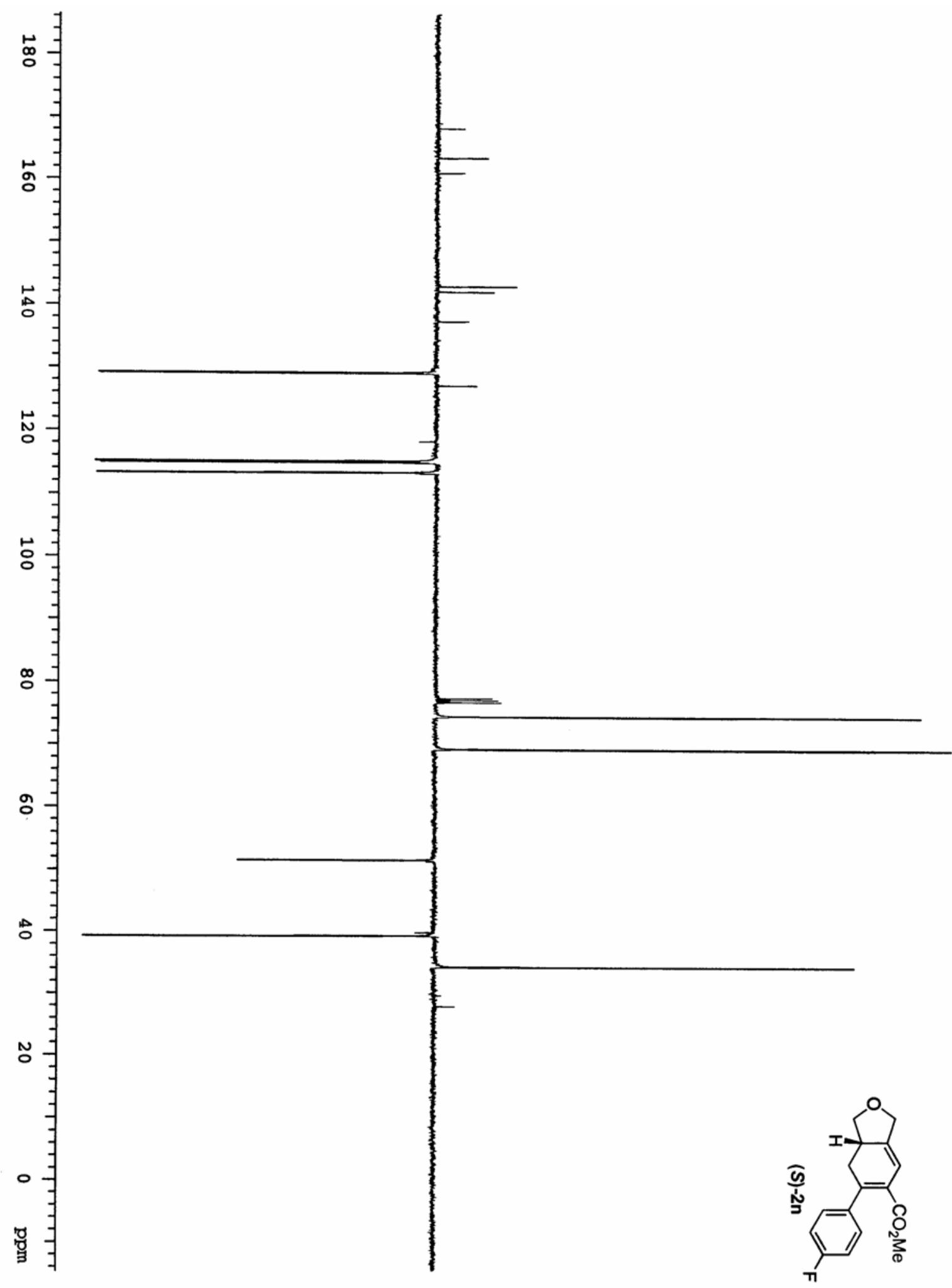




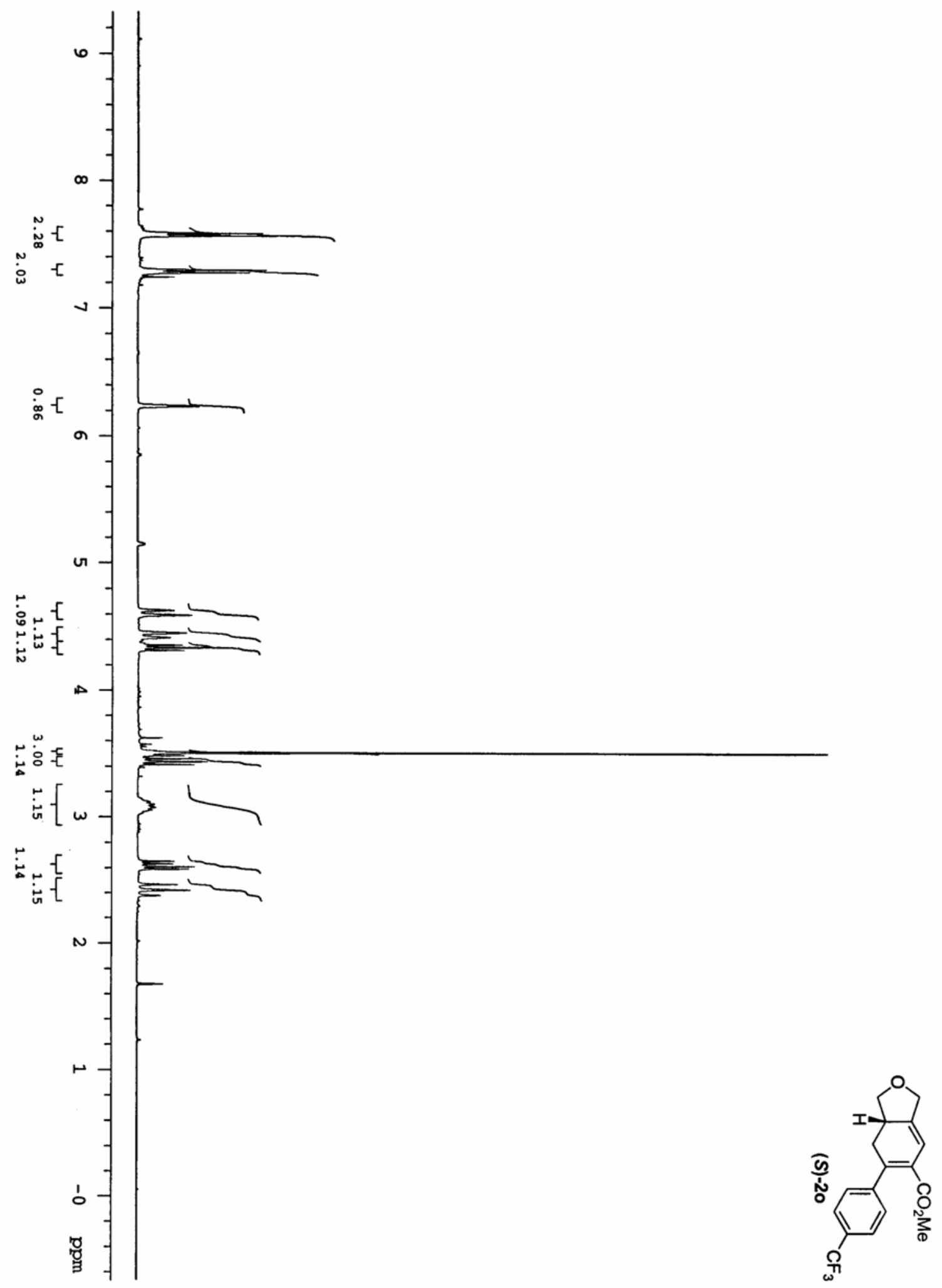




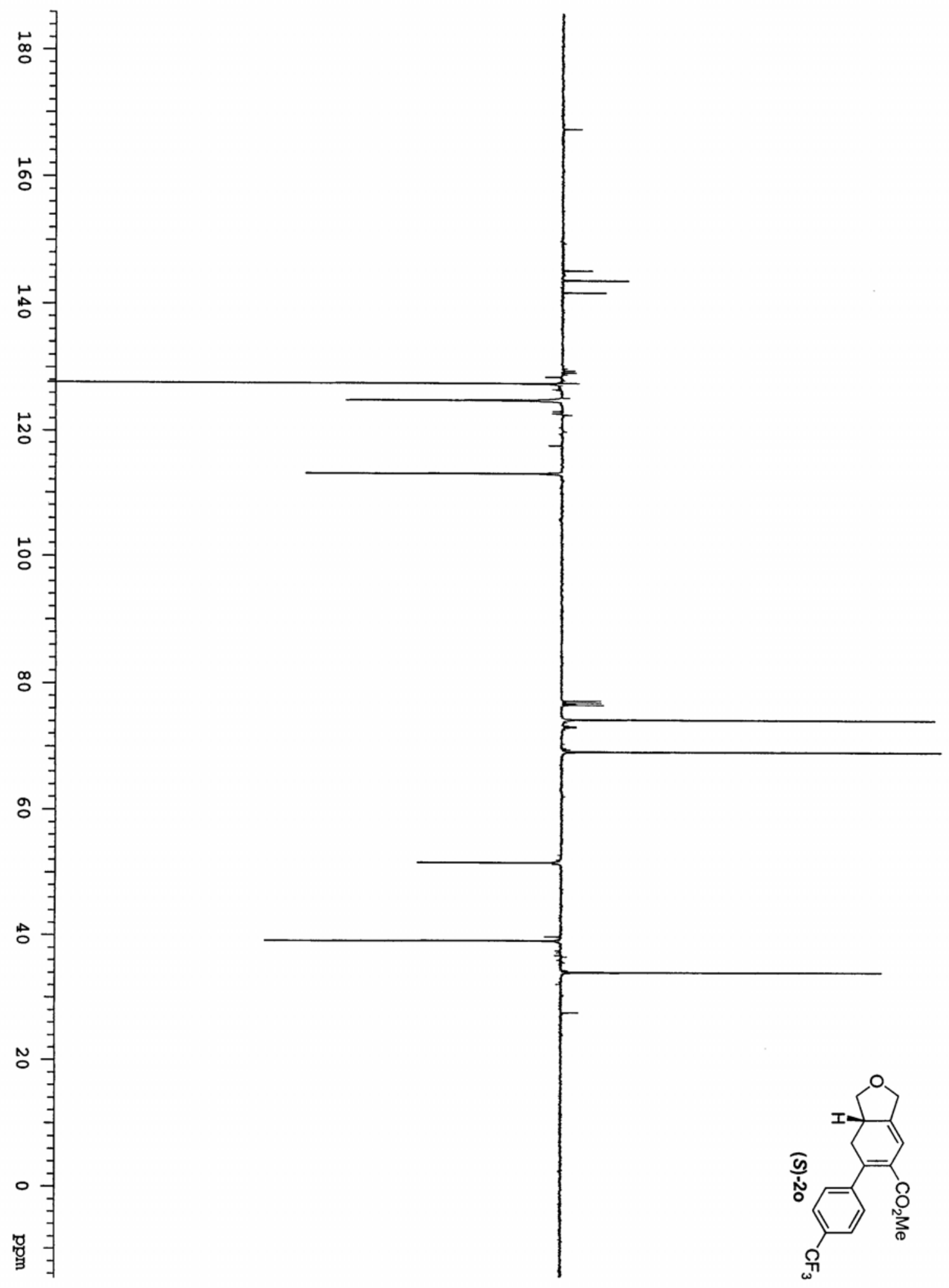




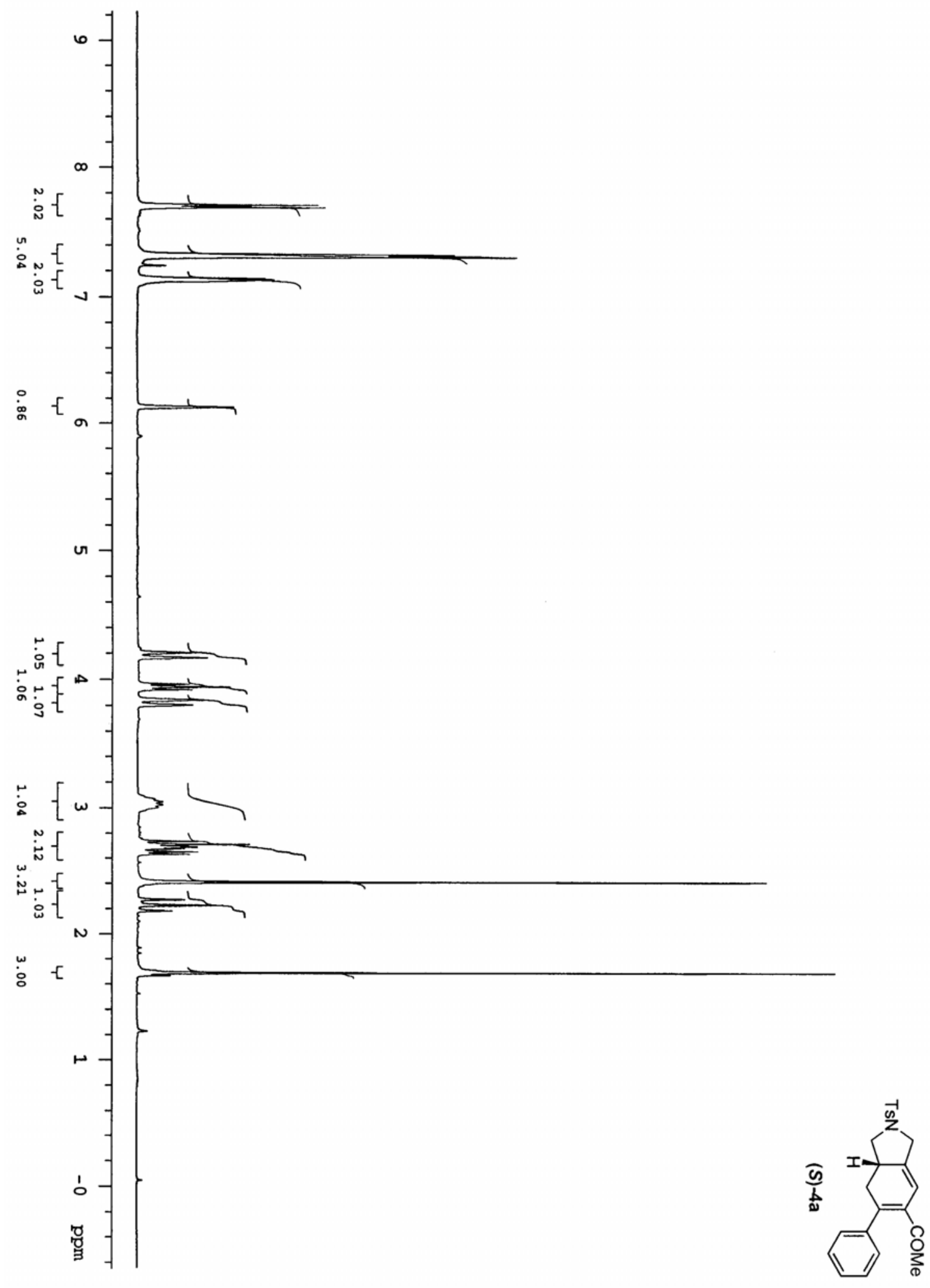




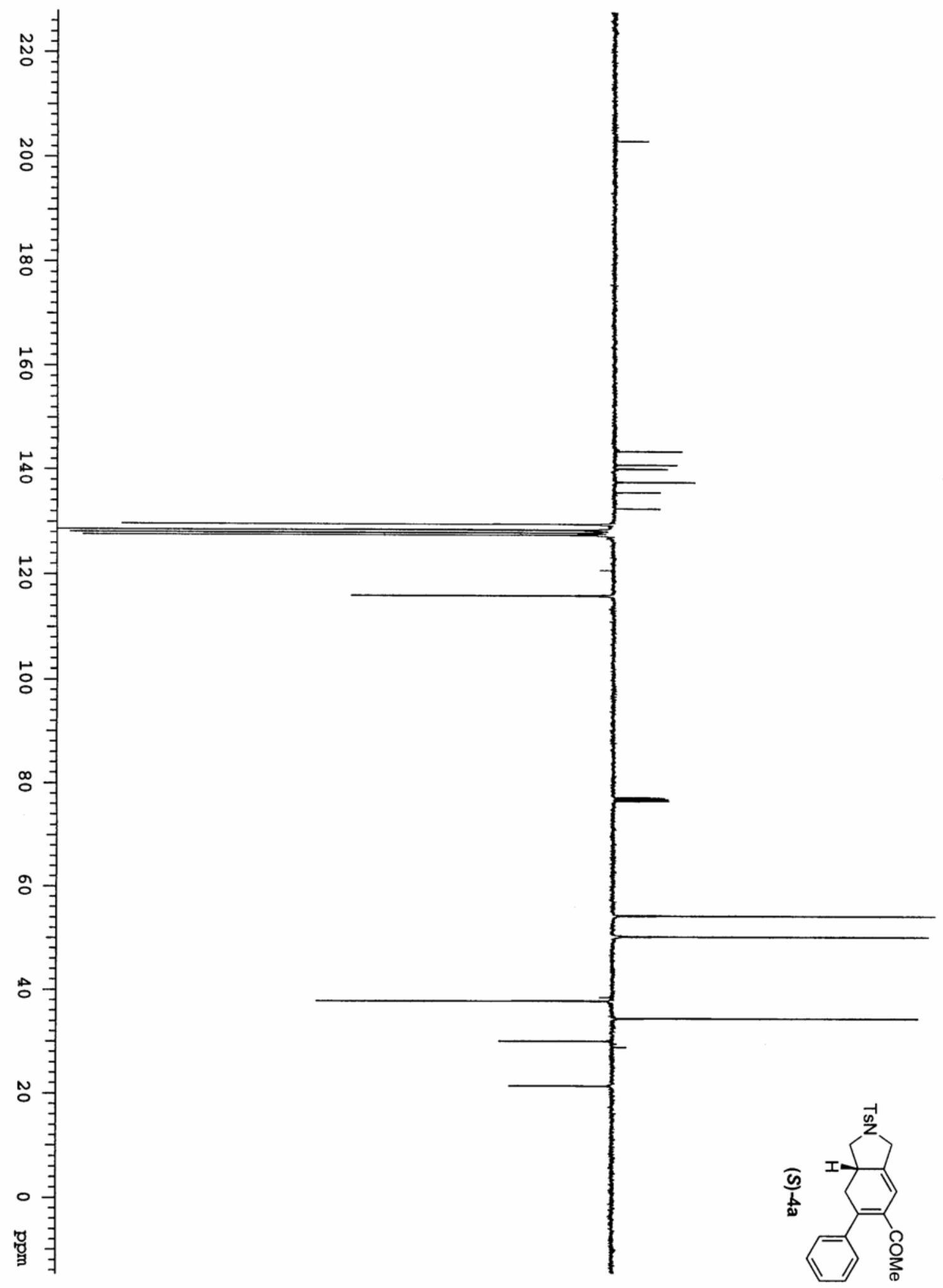




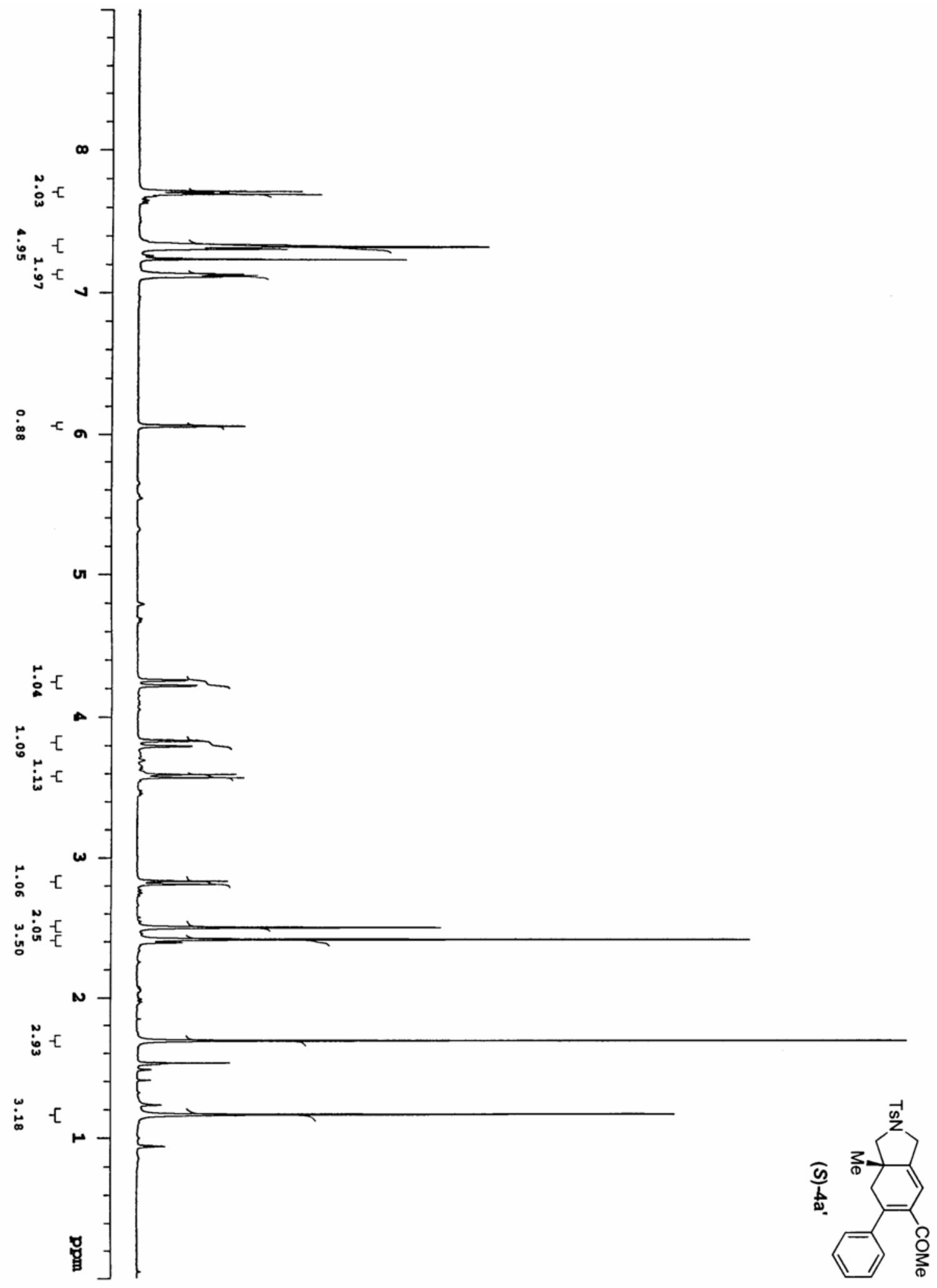




$$
H
$$




\title{
Indiana University Molecular Structure Center
}

\author{
Report 05149: $\mathrm{C}_{23} \mathrm{H}_{23} \mathrm{NO}_{4} \mathrm{~S}$ \\ Compound (S)-2a
}

by John C. Huffman

May 10, 2005

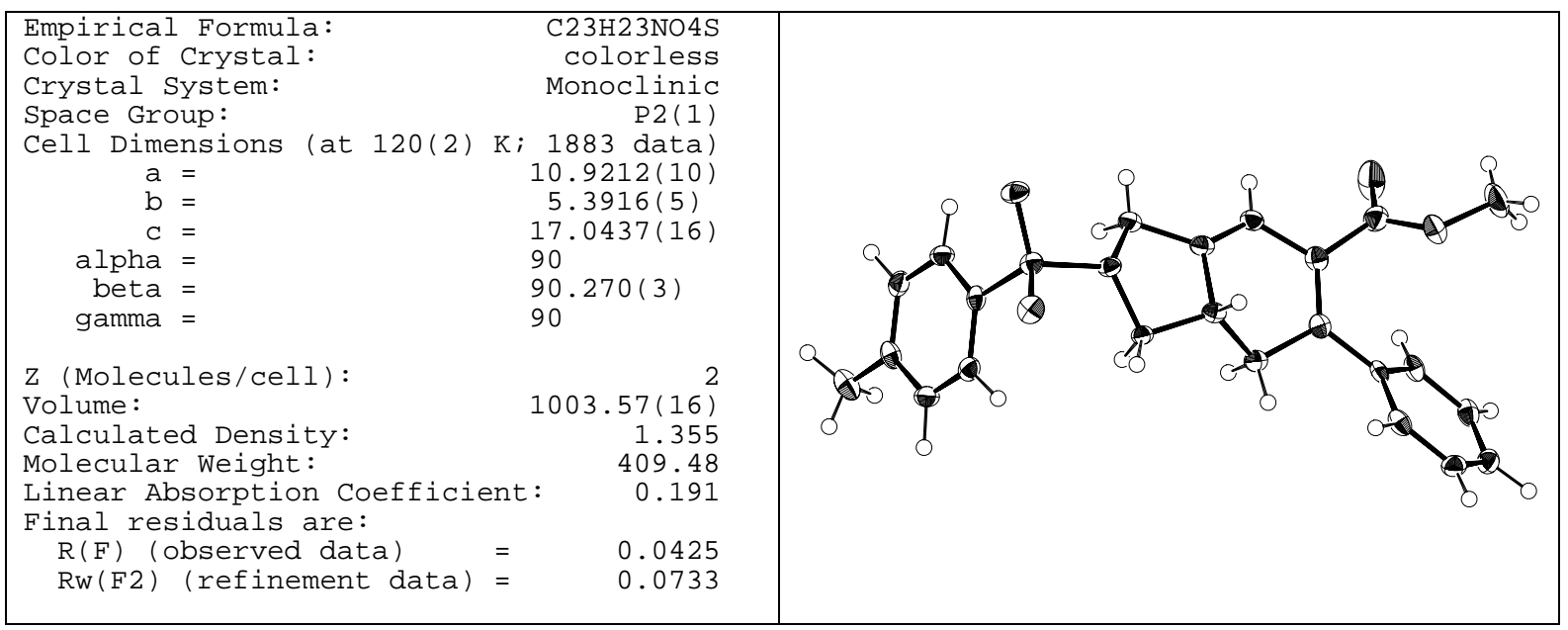

The sample was submitted by James Sawyer from the research group of Prof. P.A. Evans, Department of Chemistry, Indiana University. The crystals occur as transparent colorless rectangular plates that tend to grow in clumps. A typical crystal of approximate dimensions $0.40 \times 0.18 \times 0.15 \mathrm{~mm}$ onto the tip of a $0.1 \mathrm{~mm}$ diameter glass fiber which was subsequently mounted on a SMART6000 (Bruker) and cooled to 120(2) K.

\section{Data collection}

A preliminary set of cell constants was calculated from reflections obtained from three nearly orthogonal sets of 20 frames. The data collection was carried out using graphite monochromated Mo $\mathrm{K} \alpha$ radiation with a frame time of 30 seconds and a detector distance of $5.0 \mathrm{~cm}$. A randomly oriented region of a sphere in reciprocal space was surveyed. Three sections of 606 frames were collected with $0.30^{\circ}$ steps in $\omega$ at different $\phi$ settings with the detector set at $-43^{\circ}$ in $2 \theta$. Final cell constants were calculated from the xyz centroids of 1883 strong reflections from the actual data collection after integration (SAINT).

\section{Structure solution and refinement}

Intensity statistics and systematic absences suggested the non-centrosymmetric space group $\mathrm{P} 2{ }_{1}$ and subsequent solution and refinement confirmed this choice. The structure was solved using SHELXS-97 and refined with SHELXL-97. A direct-methods solution was calculated which provided most non-hydrogen atoms from the E-map. Full-matrix least squares / difference Fourier cycles were performed which located the remaining non-hydrogen atoms. All non-hydrogen atoms were refined with anisotropic displacement parameters. All hydrogen atoms were located in subsequent Fourier maps and included as isotropic contributors in the final cycles of refinement.

Complete data are available at

http://bl-chem-iumsc110.chem.indiana.edu/recipnet/showsample.jsp?sampleId=64531609\&sampleHistoryId=-1 